\title{
Sign conjugacy classes of the symmetric groups
}

\author{
Lucia Morotti \\ Lehrstuhl D für Mathematik \\ RWTH Aachen University \\ Aachen, Germany \\ lucia.morotti@matha.rwth-aachen.de
}

Submitted: Feb 25, 2015; Accepted: Jun 25, 2015; Published: Jul 17, 2015

Mathematics Subject Classifications: 20C30

\begin{abstract}
A conjugacy class $C$ of a finite group $G$ is a sign conjugacy class if every irreducible character of $G$ takes value 0,1 or -1 on $C$. In this paper we classify the sign conjugacy classes of the symmetric groups and thereby verify a conjecture of Olsson.
\end{abstract}

Keywords: symmetric groups; characters; partitions

\section{Introduction}

We will begin this paper by giving the definition of sign conjugacy class for an arbitrary finite group.

Definition 1.1. Let $G$ be a finite group. A conjugacy class of $G$ is a sign conjugacy class of $G$ if every irreducible character of $G$ takes values 0,1 or -1 on $C$.

Since we will be working with the symmetric group, we will consider partitions instead of conjugacy classes. A partition of $n$ is a sign partition if it is the corresponding conjugacy class of $S_{n}$ is a sign conjugacy class. An easy example of a sign partition of $n$ is $(n)$.

Definition 1.2. Define Sign to be the subsets of partitions consisting of all partitions $\left(\gamma_{1}, \ldots, \gamma_{r}\right)$ for which there exists an $s, 0 \leqslant s \leqslant r$, such that the following hold:

- $\gamma_{i}>\gamma_{i+1}+\cdots+\gamma_{r}$ for $1 \leqslant i \leqslant s$,

- $\left(\gamma_{s+1}, \ldots, \gamma_{r}\right)$ is one of the following partitions:

- (), (1, 1), $(3,2,1,1)$ or $(5,3,2,1)$,

$-(a, a-1,1)$ with $a \geqslant 2$, 


$$
\begin{aligned}
& -(a, a-1,2,1) \text { with } a \geqslant 4, \\
& -(a, a-1,3,1) \text { with } a \geqslant 5 .
\end{aligned}
$$

The name Sign for the above set is justified by the next theorem, which classifies sign partitions.

Theorem 1.3. A partition $\gamma$ is a sign partition if and only if $\gamma \in \operatorname{Sign}$.

This was first formulated by Olsson in [4] as a conjecture.

In order to prove Theorem 1.3 we will use two results from [4]. The first one of them is the following lemma (Theorem 7 of [4]).

Lemma 1.4. A sign partition cannot have repeated parts, except possibly for the part 1, which may have multiplicity 2.

In particular only partitions of the form $\left(\gamma_{1}, \ldots, \gamma_{r}\right)$ with either $\gamma_{1}>\ldots>\gamma_{r}$ or $\gamma_{1}>\ldots>\gamma_{r-2}>\gamma_{r-1}=\gamma_{r}=1$ may be sign partitions. The next lemma can also be found in [4] (Proposition 2).

Lemma 1.5. Let $\left(\gamma_{1}, \ldots, \gamma_{r}\right)$ be a partition of $n$ and let $m>n$. Then $\left(\gamma_{1}, \ldots, \gamma_{r}\right)$ is a sign partition if and only if $\left(m, \gamma_{1}, \ldots, \gamma_{r}\right)$ is a sign partition.

For any partition $\lambda=\left(\lambda_{1}, \ldots, \lambda_{k}\right)$ let $|\lambda|:=\lambda_{1}+\cdots+\lambda_{k}$. Also for $1 \leqslant i \leqslant k$ and $1 \leqslant j \leqslant \lambda_{i}$ let $h_{i, j}^{\lambda}$ denote the hook length of the node $(i, j)$ of $\lambda$. For partitions $\lambda, \mu$ with $|\lambda|=n=|\mu|$ let $\chi_{\mu}^{\lambda}$ denote the value of the irreducible character of $S_{n}$ labeled by $\lambda$ on the conjugacy class with cycle partition $\mu$.

Together with the previous lemmas, the following theorem, which will be proved in Sections 2 and 3, will allow us to prove one direction of Theorem 1.3.

Theorem 1.6. Let $\alpha=\left(\alpha_{1}, \ldots, \alpha_{h}\right)$ be a partition with $h \geqslant 3$. Assume that $\alpha_{1}>\alpha_{2}$, that $\alpha \notin \operatorname{Sign}$ and that $\left(\alpha_{2}, \ldots, \alpha_{h}\right) \in$ Sign. Then if $\alpha \neq(5,4,3,2,1)$ we can find a partition $\beta$ of $|\alpha|$ such that $\chi_{\alpha}^{\beta} \notin\{0, \pm 1\}$ and $h_{2,1}^{\beta}=\alpha_{1}$.

The other direction of Theorem 1.3 will be proved using Lemma 1.5 and the results from Section 4 , where we prove that the partitions $\left(\gamma_{s+1}, \ldots, \gamma_{r}\right)$ are sign partitions.

References about results on partitions and irreducible characters of $S_{n}$ can be found in [1] and [3].

\section{Proof of Theorem 1.6 for $\alpha_{2} \leqslant \alpha_{3}+\cdots+\alpha_{h}$}

In this section we will prove Theorem 1.6 in the case where $\alpha_{2} \leqslant \alpha_{3}+\cdots+\alpha_{h}$. Since by assumption $h \geqslant 3$ and $\left(\alpha_{2}, \ldots, \alpha_{h}\right) \in$ Sign, we have that

$$
\begin{array}{r}
\left(\alpha_{2}, \ldots, \alpha_{h}\right) \in\{(1,1),(3,2,1,1),(5,3,2,1)\} \cup\{(a, a-1,1): a \geqslant 2\} \\
\cup\{(a, a-1,2,1): a \geqslant 4\} \cup\{(a, a-1,3,1): a \geqslant 5\} .
\end{array}
$$


Also $\alpha_{1} \leqslant \alpha_{2}+\cdots+\alpha_{h}$ as $\alpha \notin$ Sign and by assumption $\alpha_{1}>\alpha_{2}$. If

$$
\begin{aligned}
\left(\alpha_{2}, \ldots, \alpha_{h}\right) \in\{ & (1,1),(3,2,1,1),(5,3,2,1)\} \cup\{(a, a-1,1): 2 \leqslant a \leqslant 4\} \\
& \cup\{(a, a-1,2,1): 4 \leqslant a \leqslant 8\} \cup\{(a, a-1,3,1): 5 \leqslant a \leqslant 10\}
\end{aligned}
$$

there are only finitely many such $\alpha$ and it can be checked that for each one of them Theorem 1.6 holds.

For $\left(\alpha_{2}, \ldots, \alpha_{h}\right)=(a, a-1,1)$ with $a \geqslant 5$ let

$$
\beta:= \begin{cases}\left(2 a, 2,1^{\alpha_{1}-2}\right), & a+2 \leqslant \alpha_{1} \leqslant 2 a-2 \text { or } \alpha_{1}=2 a, \\ (a-1, a-1, a-1,4), & \alpha_{1}=a+1, \\ \left(2 a, \alpha_{1}\right), & \alpha_{1}=2 a-1 .\end{cases}
$$

For $\left(\alpha_{2}, \ldots, \alpha_{h}\right)=(a, a-1,2,1)$ with $a \geqslant 9$ let

$$
\beta:= \begin{cases}\left(2 a+2,4,1^{\alpha_{1}-4}\right), & a+4 \leqslant \alpha_{1} \leqslant 2 a-2 \text { or } 2 a \leqslant \alpha_{1} \leqslant 2 a+2, \\ \left(2 a+2, \alpha_{1}-1,1\right), & \alpha_{1}=a+1 \\ \left(2 a+2,2,1^{\alpha_{1}-2}\right), & a+2 \leqslant \alpha_{1} \leqslant a+3, \\ \left(2 a+2, \alpha_{1}\right), & \alpha_{1}=2 a-1 .\end{cases}
$$

For $\left(\alpha_{2}, \ldots, \alpha_{h}\right)=(a, a-1,3,1)$ with $a \geqslant 11$ let

$$
\beta:= \begin{cases}\left(2 a+3,5,1^{\alpha_{1}-5}\right), & a+5 \leqslant \alpha_{1} \leqslant 2 a-2 \text { or } 2 a \leqslant \alpha_{1} \leqslant 2 a+3, \\ \left(2 a+3,2,1^{\alpha_{1}-2}\right), & \alpha_{1}=a+1 \text { or } \alpha_{1}=a+4, \\ \left(2 a+3, \alpha_{1}-2,1,1\right), & \alpha_{1}=a+2, \\ \left(2 a+3, \alpha_{1}\right), & \alpha_{1}=a+3 \text { or } \alpha_{1}=2 a-1 .\end{cases}
$$

It's easy to check that in each of the above cases $\beta$ is a partition and that $h_{2,1}^{\beta}=\alpha_{1}$. In each of the above cases in can also be proved that $\chi_{\alpha}^{\beta} \notin\{0, \pm 1\}$.

Assume that $\left(\alpha_{2}, \ldots, \alpha_{h}\right)=(a, a-1,1)$ and $a+2 \leqslant \alpha_{1} \leqslant 2 a-2$, that $\left(\alpha_{2}, \ldots, \alpha_{h}\right)=$ $(a, a-1,2,1)$ and $a+4 \leqslant \alpha_{1} \leqslant 2 a-2$ or that $\left(\alpha_{2}, \ldots, \alpha_{h}\right)=(a, a-1,3,1)$ and $a+5 \leqslant$ $\alpha_{1} \leqslant 2 a-2$. In either case $h_{1, \beta_{2}+1}=2 a-2 \geqslant \alpha_{1}$. As $h_{2,1}^{\beta}=\alpha_{1}$ it follows from the Murnaghan-Nakayama formula that

$$
\chi_{\alpha}^{\beta}=(-1)^{\alpha_{1}-\beta_{2}} \chi_{\left(\alpha_{2}, \ldots, \alpha_{h}\right)}^{\left(|\alpha|-\alpha_{1}\right)}+\chi_{\left(\alpha_{2}, \ldots, \alpha_{h}\right)}^{\left(|\alpha|-2 \alpha_{1}, \beta_{2}, 1^{\alpha_{1}-\beta_{2}}\right)} .
$$

Since by assumption

$$
\begin{aligned}
& h_{3,1}^{\left(|\alpha|-2 \alpha_{1}, \beta_{2}, 1^{\alpha_{1}-\beta_{2}}\right)}=\alpha_{1}-\beta_{2} \geqslant a, \\
& h_{1,2}^{\left(|\alpha|-2 \alpha_{1}, \beta_{2}, 1^{\alpha_{1}-\beta_{2}}\right)}=|\alpha|-2 \alpha_{1} \leqslant a-2,
\end{aligned}
$$

and $\alpha_{2}=a$, we have that

$$
\chi_{\alpha}^{\beta}=(-1)^{\alpha_{1}-\beta_{2}}+(-1)^{\alpha_{2}-1} \chi_{\left(\alpha_{3}, \ldots, \alpha_{h}\right)}^{\left(|\alpha|-2 \alpha_{1}, \beta_{2}, 1^{\left.\alpha_{1}-\beta_{2}-\alpha_{2}\right)} .\right.}
$$


By definition of $\beta$

$$
\begin{aligned}
h_{1,1}^{\left(|\alpha|-2 \alpha_{1}, \beta_{2}, 1^{\alpha_{1}-\beta_{2}-a}\right)} & =|\alpha|-2 \alpha_{1}+\alpha_{1}-\beta_{2}-\alpha_{2}+1 \\
& =\alpha_{3}+\cdots+\alpha_{h}-\left(\alpha_{4}+\cdots+\alpha_{h}+1\right)+1 \\
& =\alpha_{3} .
\end{aligned}
$$

So

$$
\chi_{\alpha}^{\beta}=(-1)^{\alpha_{1}-\beta_{2}}+(-1)^{\alpha_{2}-1+\alpha_{1}-\beta_{2}-\alpha_{2}+1} \chi_{\left(\alpha_{4}, \ldots, \alpha_{h}\right)}^{\left(\beta_{2}-1\right)}=(-1)^{\alpha_{1}-\beta_{2}} 2 .
$$

The other cases can be computed similarly.

\section{Proof of Theorem 1.6 for $\alpha_{2}>\alpha_{3}+\cdots+\alpha_{h}$}

In this section we will prove Theorem 1.6 for $\alpha_{2}>\alpha_{3}+\cdots+\alpha_{h}$. Again, from Lemma 1.5 , as $\alpha \notin$ Sign but $\left(\alpha_{2}, \ldots, \alpha_{h}\right) \in$ Sign, we have that $\alpha_{1} \leqslant \alpha_{2}+\cdots+\alpha_{h}$.

Throughout this section let $k$ be minimal such that

$$
\alpha_{k}+\cdots+\alpha_{h}<\alpha_{1}-\alpha_{2} .
$$

Since $\alpha_{1} \leqslant \alpha_{2}+\cdots+\alpha_{h}$, it follows that $4 \leqslant k \leqslant h+1$. Also define

$$
x:=\alpha_{k}+\cdots+\alpha_{h} .
$$

Theorem 3.1. Assume that the following hold:

- $\alpha \notin \operatorname{Sign},\left(\alpha_{2}, \ldots, \alpha_{h}\right) \in \operatorname{Sign}$ and $\alpha_{1}>\alpha_{2}>\alpha_{3}+\cdots+\alpha_{h}$,

- $k \leqslant h$,

- $\alpha_{1}-\alpha_{2}$ is not a part of $\alpha$,

- $\alpha_{k-1}>x$.

Then $\beta=\left(|\alpha|-\alpha_{1}, x+1,1^{\alpha_{1}-x-1}\right)$ is a partition, $h_{2,1}^{\beta}=\alpha_{1}$ and $\chi_{\alpha}^{\beta}=(-1)^{\alpha_{1}-x-1} 2$.

Proof. By definition and by assumption

$$
|\alpha|-\alpha_{1}=\alpha_{2}+\cdots+\alpha_{h} \geqslant \alpha_{1} \geqslant x+1,
$$

from which follows that $\beta$ is a partition. Also clearly $h_{2,1}^{\beta}=\alpha_{1}$. We will now prove that $\chi_{\alpha}^{\beta}=(-1)^{\alpha_{1}-x-1} 2$.

Assume first that $2 \alpha_{1}+x>|\alpha|$. Then

$$
2=|\alpha|-\alpha_{1}-\left(\alpha_{2}+\cdots+\alpha_{h}\right)+2 \leqslant|\alpha|-2 \alpha_{1}+2 \leqslant x+1
$$

and so

$$
h_{1,|\alpha|-2 \alpha_{1}+2}^{\beta}=|\alpha|-\alpha_{1}+2-\left(|\alpha|-2 \alpha_{1}+2\right)=\alpha_{1} .
$$


It follows that

$$
\chi_{\alpha}^{\beta}=(-1)^{\alpha_{1}-x-1} \chi_{\left(\alpha_{2}, \ldots, \alpha_{h}\right)}^{\left(|\alpha|-\alpha_{1}\right)}-\chi_{\left(\alpha_{2}, \ldots, \alpha_{h}\right)}^{\delta}=(-1)^{\alpha_{1}-x-1}-\chi_{\left(\alpha_{2}, \ldots, \alpha_{h}\right)}^{\delta},
$$

where $\delta:=\left(x,|\alpha|-2 \alpha_{1}+1,1^{\alpha_{1}-x-1}\right)$. So it is enough to prove that $\chi_{\left(\alpha_{2}, \ldots, \alpha_{h}\right)}^{\delta}=(-1)^{\alpha_{1}-x}$. As $h_{1,2}^{\delta} \leqslant x<\alpha_{k-1}<\alpha_{2}$ by assumption, we have that

$$
\chi_{\left(\alpha_{2}, \ldots, \alpha_{h}\right)}^{\delta}=(-1)^{\alpha_{2}-1} \chi_{\left(\alpha_{3}, \ldots, \alpha_{h}\right)}^{\epsilon},
$$

where $\epsilon:=\left(x,|\alpha|-2 \alpha_{1}+1,1^{\alpha_{1}-\alpha_{2}-x-1}\right.$ ) (as by definition of $x, \alpha_{1}-\alpha_{2}>x$, so that $\epsilon$ is a partition). By minimality of $k$,

$$
|\epsilon|<2 x+\alpha_{1}-\alpha_{2}-x \leqslant 2 x+\alpha_{k-1} .
$$

Also, as $\left(\alpha_{2}, \ldots, \alpha_{h}\right) \in$ Sign and $k-2 \geqslant 2$,

$$
\alpha_{3}+\cdots+\alpha_{h}=|\epsilon|<2\left(\alpha_{k}+\cdots+\alpha_{h}\right)+\alpha_{k-1}<\alpha_{k-2}+\cdots+\alpha_{h}
$$

and then $k-2<3$. Since $k \geqslant 4$ it follows that $k=4$. As by induction $\alpha_{3}>x$,

$$
\chi_{\left(\alpha_{2}, \ldots, \alpha_{h}\right)}^{\delta}=(-1)^{\alpha_{2}-1} \chi_{\left(\alpha_{3}, \ldots, \alpha_{h}\right)}^{\epsilon}=(-1)^{\alpha_{2}-1+\alpha_{1}-\alpha_{2}-x-1} \chi_{\left(\alpha_{4}, \ldots, \alpha_{h}\right)}^{(x)}=(-1)^{\alpha_{1}-x}
$$

and then the theorem holds in this case.

Assume now that $2 \alpha_{1}+x<|\alpha|$. Then

$$
x+1<|\alpha|-2 \alpha_{1}+1 \leqslant|\alpha|-\alpha_{1}
$$

and so

$$
h_{1,|\alpha|-2 \alpha_{1}+1}^{\beta}=|\alpha|-\alpha_{1}+1-\left(|\alpha|-2 \alpha_{1}+1\right)=\alpha_{1} .
$$

By definition $\alpha_{2} \leqslant \alpha_{1}-x-1$ and by assumption $\alpha_{2}>\alpha_{3}+\cdots+\alpha_{h}$, so that any partition of $\alpha_{2}+\cdots+\alpha_{h}$ has at most one hook of length $\alpha_{2}$. So

$$
\begin{aligned}
\chi_{\alpha}^{\beta} & =(-1)^{\alpha_{1}-x-1} \chi_{\left(\alpha_{2}, \ldots, \alpha_{h}\right)}^{\left(|\alpha|-\alpha_{1}\right)}+\chi_{\left(\alpha_{2}, \ldots, \alpha_{h}\right)}^{\left(|\alpha|-2 \alpha_{1}, x+1,1^{\alpha_{1}-x-1}\right)} \\
& =(-1)^{\alpha_{1}-x-1}+(-1)^{\alpha_{2}-1} \chi_{\left(\alpha_{3}, \ldots, \alpha_{h}\right)}^{\lambda},
\end{aligned}
$$

where $\lambda=\left(|\alpha|-2 \alpha_{1}, x+1,1^{\alpha_{1}-\alpha_{2}-x-1}\right)$. So it is enough to prove that $\chi_{\left(\alpha_{3}, \ldots, \alpha_{h}\right)}^{\lambda}=$ $(-1)^{\alpha_{1}-\alpha_{2}-x}$.

First assume that $\alpha_{k-1}>\alpha_{1}-\alpha_{2}$. Then

$$
h_{2,1}^{\lambda}=\alpha_{1}-\alpha_{2}<\alpha_{j}
$$

for $3 \leqslant j \leqslant k-1$ and

$$
h_{1, x+2}^{\lambda}=|\lambda|-x-1-\alpha_{1}+\alpha_{2} \geqslant|\lambda|-\alpha_{k-1}-\cdots-\alpha_{h}=\alpha_{3}+\cdots+\alpha_{k-2}
$$


if $x+2 \leqslant \lambda_{1}$. If $\lambda_{1}=x+1$ then

$$
|\lambda|=x+\alpha_{1}-\alpha_{2}+1 \leqslant \alpha_{k-1}+\cdots+\alpha_{h} \leqslant \alpha_{3}+\cdots+\alpha_{h}=|\lambda|
$$

and so in this case $k=4$. In either case

$$
\begin{aligned}
\chi_{\left(\alpha_{3}, \ldots, \alpha_{h}\right)}^{\lambda} & =\chi_{\left(\alpha_{k-1}, \ldots, \alpha_{h}\right)}^{\left(\alpha_{k-1}-\alpha_{1}+\alpha_{2}+x, x+1,1^{\alpha_{1}-\alpha_{2}-x-1}\right)} \\
& =(-1)^{\alpha_{1}-\alpha_{2}-x} \chi_{\left(\alpha_{k}, \ldots, \alpha_{h}\right)}^{(x)} \\
& =(-1)^{\alpha_{1}-\alpha_{2}-x}
\end{aligned}
$$

and so the theorem holds also in this case.

Now assume that $\alpha_{k-1}<\alpha_{1}-\alpha_{2}$. Then $k \geqslant 5$ (otherwise $\alpha_{1}>\alpha_{2}+\cdots+\alpha_{h}$ ) and

$$
\alpha_{k-1}+x=\alpha_{k-1}+\cdots+\alpha_{h} \geqslant \alpha_{1}-\alpha_{2}
$$

by definition of $k$. Since $\alpha_{1}-\alpha_{2}-x-1<\alpha_{k-1}$ by minimality of $k$ and since by assumption $x<\alpha_{k-1}$ and $\alpha_{1}-\alpha_{2}$ is not a part of $\alpha$, it follows similarly to the previous case that

$$
\chi_{\left(\alpha_{3}, \ldots, \alpha_{h}\right)}^{\lambda}=\chi_{\left(\alpha_{k-2}, \ldots, \alpha_{h}\right)}^{\mu},
$$

where $\mu:=\left(\alpha_{k-2}+\alpha_{k-1}-\alpha_{1}+\alpha_{2}+x, x+1,1^{\alpha_{1}-\alpha_{2}-x-1}\right)$. As

$$
2 \leqslant \alpha_{k-1}-\alpha_{1}+\alpha_{2}+x+2 \leqslant x+1
$$

and so

$$
h_{1, \alpha_{k-1}-\alpha_{1}+\alpha_{2}+x+2}^{\mu}=\alpha_{k-2}+\alpha_{k-1}-\alpha_{1}+\alpha_{2}+x+2-\left(\alpha_{k-1}-\alpha_{1}+\alpha_{2}+x+2\right)=\alpha_{k-2} .
$$

From $\alpha_{1}-\alpha_{2}$ not being a part of $\alpha$ and

$$
x, \alpha_{1}-\alpha_{2}-x-1<\alpha_{k-1}<\alpha_{k-2}
$$

it follows that

$$
\chi_{\left(\alpha_{k-2}, \ldots, \alpha_{h}\right)}^{\mu}=-\chi_{\left(\alpha_{k-1}, \ldots, \alpha_{h}\right)}^{\nu}=(-1)^{\alpha_{1}-\alpha_{2}-x},
$$

with $\nu=\left(x, \alpha_{k-1}-\alpha_{1}+\alpha_{2}+x+1,1^{\alpha_{1}-\alpha_{2}-x-1}\right)$, and so the theorem holds also in this case.

At last assume that $2 \alpha_{1}+x=|\alpha|$. Then

$$
\alpha_{1}=|\alpha|-\alpha_{1}-x=\alpha_{2}+\cdots+\alpha_{k-1} .
$$

By definition of $k$ we then have that

$$
\alpha_{3}+\cdots+\alpha_{k-1}=\alpha_{1}-\alpha_{2} \leqslant \alpha_{k-1}+\cdots+\alpha_{h}
$$

and so

$$
\alpha_{3}+\cdots+\alpha_{k-2} \leqslant \alpha_{k}+\cdots+\alpha_{h} .
$$

If $k \geqslant 5$ then $k-2 \geqslant 3$ and then $\alpha_{k-2} \leqslant \alpha_{k}+\cdots+\alpha_{h}$. This gives a contradiction with $\left(\alpha_{2}, \ldots, \alpha_{h}\right) \in \operatorname{Sign}$. So $k=4$ and then $\alpha_{1}-\alpha_{2}=\alpha_{3}$ is a part of $\alpha$, which contradicts the assumptions. 
Theorem 3.2. Assume that the following hold:

- $\alpha \notin \operatorname{Sign},\left(\alpha_{2}, \ldots, \alpha_{h}\right) \in \operatorname{Sign}$ and $\alpha_{1}>\alpha_{2}>\alpha_{3}+\cdots+\alpha_{h}$,

- $k \leqslant h$,

- $\alpha_{1}-\alpha_{2}$ is not a part of $\alpha$,

- $\alpha_{k-1} \leqslant x$,

- none of the following holds:

$$
\begin{aligned}
& -\left(\alpha_{k-1}, \ldots, \alpha_{h}\right)=(3,2,1,1) \text { and } \alpha_{1}=\alpha_{2}+\alpha_{k-1}+\cdots+\alpha_{h}, \\
& -\left(\alpha_{k-1}, \ldots, \alpha_{h}\right)=(5,3,2,1) \text { and } \alpha_{1}=\alpha_{2}+\alpha_{k-1}+\cdots+\alpha_{h}, \\
& -\left(\alpha_{k-1}, \ldots, \alpha_{h}\right)=(a, a-1,1) \text { with } a \geqslant 2 \text { and } \alpha_{1}=\alpha_{2}+\alpha_{k-1}+\cdots+\alpha_{h}-1, \\
& -\left(\alpha_{k-1}, \ldots, \alpha_{h}\right)=(a, a-1,2,1) \text { with } a \geqslant 4 \text { and } \alpha_{1}=\alpha_{2}+\alpha_{k-1}+\cdots+\alpha_{h}-3, \\
& -\left(\alpha_{k-1}, \ldots, \alpha_{h}\right)=(a, a-1,3,1) \text { with } a \geqslant 5 \text { and } \alpha_{1}=\alpha_{2}+\alpha_{k-1}+\cdots+\alpha_{h}-4 .
\end{aligned}
$$

Then $\beta=\left(|\alpha|-\alpha_{1}, x+1,1^{\alpha_{1}-x-1}\right)$ is a partition, $h_{2,1}^{\beta}=\alpha_{1}$ and $\chi_{\alpha}^{\beta}=(-1)^{\alpha_{1}-x-1} 2$.

Proof. As in the previous theorem we have that $2 \alpha_{1}+x \neq|\alpha|$, since $\alpha_{1}-\alpha_{2}$ is not a part of $\alpha$.

Assume first that $2 \alpha_{1}+x>|\alpha|$. From the proof of the previous theorem $\left(\alpha_{2}>x\right.$ since $\left(\alpha_{2}, \ldots, \alpha_{h}\right) \in$ Sign $)$, it is enough to prove that $\chi_{\left(\alpha_{3}, \ldots, \alpha_{h}\right)}^{\epsilon}=(-1)^{\alpha_{1}-\alpha_{2}-x-1}$, where $\epsilon=\left(x,|\alpha|-2 \alpha_{1}+1,1^{\alpha_{1}-\alpha_{2}-x-1}\right)$. In this case it holds $k=4$ as in the previous theorem.

Assume now that $2 \alpha_{1}+x<|\alpha|$. Since $\alpha_{k-1} \leqslant x<\alpha_{1}-\alpha_{2}$ we have that $\alpha_{k-1}<$ $\alpha_{1}-\alpha_{2}$. As $\alpha_{1}-\alpha_{2}$ is not a part of $\alpha$ it is enough, from the proof of the previous theorem, to prove that $x<\alpha_{j}$ for $j \leqslant k-2$ and that $\chi_{\left(\alpha_{k-1}, \ldots, \alpha_{h}\right)}^{\nu}=(-1)^{\alpha_{1}-\alpha_{2}-x-1}$, where $\nu=\left(x, \alpha_{k-1}-\alpha_{1}+\alpha_{2}+x+1,1^{\alpha_{1}-\alpha_{2}-x-1}\right)$. In order to prove that $x<\alpha_{j}$ for $j \leqslant k-2$, it is enough to prove it for $j=k-2$. As $k \geqslant 4$, so that $k-2 \geqslant 2$, and $\left(\alpha_{2}, \ldots, \alpha_{h}\right) \in \operatorname{Sign}$, we have that $x=\alpha_{k}+\cdots+\alpha_{h}<\alpha_{k-2}$.

In either case it is then enough to prove that $\chi_{\left(\alpha_{k-1}, \ldots, \alpha_{h}\right)}^{\lambda_{y}}=(-1)^{y}$ for $\lambda_{y}=\left(x, \alpha_{k-1}-\right.$ $\left.y, 1^{y}\right), y=\alpha_{1}-\alpha_{2}-x-1$. Notice that $0 \leqslant y \leqslant \alpha_{k-1}-1$, since $\lambda_{y}$ is a partition.

Clearly $h_{2,1}^{\lambda_{y}}=\alpha_{k-1}$. If this is the only $\alpha_{k-1}$-hook of $\lambda$, then it is easy to see that $\chi_{\left(\alpha_{k-1}, \ldots, \alpha_{h}\right)}^{\lambda_{y}}=(-1)^{y}$. Else, due to hook lengths being decreasing along both the rows and the columns, $\lambda_{y}$ has exactly $2 \alpha_{k-1}$-hooks and there exists $2 \leqslant j \leqslant x$ with $h_{1, j}^{\lambda_{y}}=\alpha_{k-1}$.

As $\alpha_{k-1} \leqslant x$ by assumption

$$
\begin{aligned}
\left(\alpha_{k-1}, \ldots, \alpha_{h}\right) \in & \{(1,1),(3,2,1,1),(5,3,2,1)\} \cup\{(a, a-1,1): a \geqslant 2\} \\
& \cup\{(a, a-1,2,1): a \geqslant 4\} \cup\{(a, a-1,3,1): a \geqslant 5\} .
\end{aligned}
$$

If $\left(\alpha_{k-1}, \ldots, \alpha_{h}\right)=(1,1)$ then $x=1<2$, so no such $j$ exists.

If $\left(\alpha_{k-1}, \ldots, \alpha_{h}\right)=(3,2,1,1)$ then $\lambda_{y} \in\{(4,3),(4,1,1,1)\}$ if such a $j$ exists, and so $y=0$ or $y=2$ respectively. The second case would imply $\alpha_{1}-\alpha_{2}-x=3$, which would contradict the assumption. As $\chi_{(3,2,1,1)}^{(4,3)}=1=(-1)^{0}$ the theorem holds in this case. 
If $\left(\alpha_{k-1}, \ldots, \alpha_{h}\right)=(5,3,2,1)$ and there exists such a $j$ then

$$
\lambda_{y} \in\left\{(6,5),(6,4,1),(6,3,1,1),\left(6,1^{5}\right)\right\}
$$

and then $y=0, y=1, y=2$ or $y=4$ respectively. In the last case $\alpha_{1}-\alpha_{2}-x=5$, which contradicts the assumption. In the other cases $\chi_{(5,3,2,1)}^{(6,5)}=1=(-1)^{0}, \chi_{(5,3,2,1)}^{(6,4,1)}=$ $-1=(-1)^{1}$ and $\chi_{(5,3,2,1)}^{(6,3,1,1)}=1=(-1)^{2}$ and so the theorem holds also in this case.

If $\left(\alpha_{k-1}, \ldots, \alpha_{h}\right)=(a, a-1,1)$ then there exists such a $j$ if and only if $0 \leqslant y \leqslant \alpha_{k-1}-2$. If $y=\alpha_{k-1}-2$ then $\alpha_{1}-\alpha_{2}-x=\alpha_{k-1}-1$ which contradicts the assumption. In the other cases

$$
\chi_{\left(\alpha_{k-1}, \ldots, \alpha_{h}\right)}^{\lambda_{y}}=\chi_{(a, a-1,1)}^{\left(a, a-y, 1^{y}\right)}=(-1)^{y} \chi_{(a-1,1)}^{(a)}-\chi_{(a-1,1)}^{\left(a-y-1,1^{y+1}\right)}=(-1)^{y},
$$

since $a-y-2, y+1 \geqslant 1$, so that also $a-y-2, y+1<a-1$. In particular the theorem holds in this case.

If $\left(\alpha_{k-1}, \ldots, \alpha_{h}\right)=(a, a-1,2,1)$ then there exists such a $j$ if and only if $y \neq \alpha_{k-1}-3$. For $y=\alpha_{k-1}-4$ we have that $\alpha_{1}-\alpha_{2}-x=\alpha_{k-1}-3$, which contradicts the assumptions.

For $0 \leqslant y \leqslant \alpha_{k-1}-5$ then $j=4$ as $\alpha_{k-1}-y>4$, so that

$$
h_{1,4}^{\lambda_{y}}=a+2+2-4=a .
$$

So

$$
\chi_{\left(\alpha_{k-1}, \ldots, \alpha_{h}\right)}^{\lambda_{y}}=\chi_{(a, a-1,2,1)}^{\left(a+2, a-y, 1^{y}\right)}=(-1)^{y} \chi_{(a-1,2,1)}^{(a+2)}-\chi_{(a-1,2,1)}^{\left(a-y-1,3,1^{y}\right)}=(-1)^{y}-\chi_{(a-1,2,1)}^{\left(a-y-1,3,1^{y}\right)}
$$

and

$$
\chi_{(a-1,2,1)}^{\left(a-y-1,3,1^{y}\right)}= \begin{cases}0 & y \neq 0 \\ -\chi_{(2,1)}^{(2,1)}=0 & y=0,\end{cases}
$$

as

$$
\begin{aligned}
h_{1,1}^{\left(a-y-1,3,1^{y}\right)} & =a \\
h_{2,1}^{\left(a-y-1,3,1^{y}\right)} & =y+3<a-1, \\
h_{1,2}^{\left(a-y-1,3,1^{y}\right)} & =a-y-1 \leqslant a-1,
\end{aligned}
$$

since $0 \leqslant y \leqslant \alpha_{k-1}-5=a-5$. In particular $\chi_{\left(\alpha_{k-1}, \ldots, \alpha_{h}\right)}^{\lambda_{y}}=(-1)^{y}$.

For $\alpha_{k-1}-2 \leqslant y \leqslant \alpha_{k-1}-1$ then $j=3$ as $\alpha_{k-1}-y \leqslant 2$, so that

$$
h_{1,3}^{\lambda_{y}}=a+2+1-3=a \text {. }
$$

It follows that

$$
\chi_{\left(\alpha_{k-1}, \ldots, \alpha_{h}\right)}^{\lambda_{y}}=\chi_{(a, a-1,2,1)}^{\left(a+2, a-y, 1^{y}\right)}=(-1)^{y} \chi_{(a-1,2,1)}^{(a+2)}+\chi_{(a-1,2,1)}^{\left(2, a-y, 1^{y}\right)}=(-1)^{y}+\chi_{(a-1,2,1)}^{\left(2, a-y, 1^{y}\right)} .
$$

As

$$
\chi_{(a-1,2,1)}^{\left(2, a-y, 1^{y}\right)}= \begin{cases}\chi_{(a-1,2,1)}^{\left(2,2,1^{a-2}\right)}=0 & y=\alpha_{k-1}-2, \\ \chi_{(a-1,2,1)}^{\left(2,1^{a}\right)}=(-1)^{a-2} \chi_{(2,1)}^{(2,1)}=0 & y=\alpha_{k-1}-1,\end{cases}
$$


as $a \geqslant 4$. In particular also in this case $\chi_{\left(\alpha_{k-1}, \ldots, \alpha_{h}\right)}^{\lambda_{y}}=(-1)^{y}$.

If $\left(\alpha_{k-1}, \ldots, \alpha_{h}\right)=(a, a-1,3,1)$ then there exists such a $j$ if and only if $y \neq \alpha_{k-1}-4$. If $y=\alpha_{k-1}-5$ then $\alpha_{1}-\alpha_{2}-x=\alpha_{k}-4$, in contradiction to the assumption.

For $0 \leqslant y \leqslant \alpha_{k-1}-6$ then $j=5$ as $\alpha_{k-1}-y>5$ and then

$$
h_{1,5}^{\lambda_{y}}=a+3+2-5=a .
$$

So

$$
\chi_{\left(\alpha_{k-1}, \ldots, \alpha_{h}\right)}^{\lambda_{y}}=\chi_{(a, a-1,3,1)}^{\left(a+3, a-y, 1^{y}\right)}=(-1)^{y} \chi_{(a-1,3,1)}^{(a+3)}-\chi_{(a-1,3,1)}^{\left(a-y-1,4,1^{y}\right)}=(-1)^{y}-\chi_{(a-1,3,1)}^{\left(a-y-1,3,1^{y}\right)}
$$

and

$$
\chi_{(a-1,3,1)}^{\left(a-y-1,41^{1}\right)}= \begin{cases}0 & y \neq 0, \\ -\chi_{(3,1)}^{(3,1)}=0 & y=0,\end{cases}
$$

as

$$
\begin{aligned}
h_{1,1}^{\left(a-y-1,4,1^{y}\right)} & =a, \\
h_{2,1}^{\left(a-y-1,4,1^{y}\right)} & =y+4<a-1, \\
h_{1,2}^{\left(a-y-1,4,1^{y}\right)} & =a-y-1 \leqslant a-1,
\end{aligned}
$$

since $0 \leqslant y \leqslant \alpha_{k-1}-6=a-6$. In particular $\chi_{\left(\alpha_{k-1}, \ldots, \alpha_{h}\right)}^{\lambda_{y}}=(-1)^{y}$.

For $\alpha_{k-1}-3 \leqslant y \leqslant \alpha_{k-1}-1$ then $j=4$ as $\alpha_{k-1}-y \leqslant 3$, so that

$$
h_{1,4}^{\lambda_{y}}=a+3+1-4=a \text {. }
$$

Then

$$
\chi_{\left(\alpha_{k-1}, \ldots, \alpha_{h}\right)}^{\lambda_{y}}=\chi_{(a, a-1,3,1)}^{\left(a+3, a-y, 1^{y}\right)}=(-1)^{y} \chi_{(a-1,3,1)}^{(a+3)}+\chi_{(a-1,3,1)}^{\left(3, a-y, 1^{y}\right)}=(-1)^{y}+\chi_{(a-1,3,1)}^{\left(3, a-y, 1^{y}\right)} .
$$

As

$$
\chi_{(a-1,3,1)}^{\left(3, a-y, 1^{y}\right)}= \begin{cases}\chi_{(a-1,3,1)}^{\left(3,3,1^{a-3}\right)}=0 & y=\alpha_{k-1}-3, \\ \chi_{(a, 1,1,1,2)}^{(3,-1)}=0 & y=\alpha_{k-1}-2, \\ \chi_{(a-1,3,1)}^{\left(3,1^{a}\right)}=(-1)^{a-2} \chi_{(3,1)}^{(3,1)}=0 & y=\alpha_{k-1}-1,\end{cases}
$$

since $a \geqslant 5$ it follows that also in this case $\chi_{\left(\alpha_{k-1}, \ldots, \alpha_{h}\right)}^{\lambda_{y}}=(-1)^{y}$.

Theorem 3.3. Assume that the following hold:

- $\alpha \notin$ Sign, $\left(\alpha_{2}, \ldots, \alpha_{h}\right) \in \operatorname{Sign}$ and $\alpha_{1}>\alpha_{2}>\alpha_{3}+\cdots+\alpha_{h}$,

- $k \leqslant h$,

- $\alpha_{1}-\alpha_{2}$ is not a part of $\alpha$,

- $\left(\alpha_{k-1}, \ldots, \alpha_{h}\right) \in\{(3,2,1,1),(5,3,2,1)\}$, 
- $\alpha_{1}=\alpha_{2}+\alpha_{k-1}+\cdots+\alpha_{h}$.

Let $c$ equal to 3 if $\left(\alpha_{k-1}, \ldots, \alpha_{h}\right)=(3,2,1,1)$ or equal to 6 if $\left(\alpha_{k-1}, \ldots, \alpha_{h}\right)=(5,3,2,1)$.

Then $\beta:=\left(|\alpha|-\alpha_{1}, \alpha_{1}-c, 1^{c}\right)$ is a partition with $h_{2,1}^{\beta}=\alpha_{1}$ and $\chi_{\alpha}^{\beta}=(-1)^{c} 2$.

Proof. Since $c<\alpha_{2}<\alpha_{1}<\alpha_{2}+\cdots+\alpha_{h}=|\alpha|-\alpha_{1}$ by assumption on $\alpha$, it follows that $\beta$ is a partition. Clearly $h_{2,1}^{\beta}=\alpha_{1}$.

Also, from

$$
2 \leqslant \alpha_{3}+\cdots+\alpha_{k-2}+2<\alpha_{3}+\cdots+\alpha_{h}-c<\alpha_{1}-c
$$

we have that

$$
\begin{aligned}
h_{1, \alpha_{3}+\cdots+\alpha_{k-2}+2}^{\beta} & =|\alpha|-\alpha_{1}+2-\left(\alpha_{3}+\cdots+\alpha_{k-2}+2\right) \\
& =\alpha_{2}+\cdots+\alpha_{h}-\alpha_{3}-\cdots-\alpha_{k-2} \\
& =\alpha_{2}+\alpha_{k-1}+\cdots+\alpha_{h} \\
& =\alpha_{1} .
\end{aligned}
$$

If $\left(\alpha_{k-1}, \ldots, \alpha_{h}\right)=(3,2,1,1)$ let $d=3$. If instead $\left(\alpha_{k-1}, \ldots, \alpha_{h}\right)=(5,3,2,1)$ let $d=4$. Notice that $c+d=\alpha_{k-1}+\cdots+\alpha_{h}-1$. Then by assumption

$$
\alpha_{1}-c=\alpha_{2}+\alpha_{k-1}+\cdots+\alpha_{h}-c=\alpha_{2}+d+1 .
$$

It follows that

$$
\chi_{\alpha}^{\beta}=(-1)^{c} \chi_{\left(\alpha_{2}, \ldots, \alpha_{h}\right)}^{\left(|\alpha|-\alpha_{1}\right)}-\chi_{\left(\alpha_{2}, \ldots, \alpha_{h}\right)}^{\delta}=(-1)^{c}-\chi_{\left(\alpha_{2}, \ldots, \alpha_{h}\right)}^{\delta}
$$

where $\delta=\left(\alpha_{2}+d, \alpha_{3}+\cdots+\alpha_{k-2}+1,1^{c}\right)$.

Assume first that $k=4$. Then $\alpha_{3}+\cdots+\alpha_{k-2}=0$ and so, as $c+1<\alpha_{2}$,

$$
\chi_{\left(\alpha_{2}, \ldots, \alpha_{h}\right)}^{\delta}=\chi_{\left(\alpha_{k-1}, \ldots, \alpha_{h}\right)}^{\left(d, 1^{c+1}\right)}=(-1)^{c-1}
$$

(the last equality follows from $\left(\alpha_{k-1}, \ldots, \alpha_{h}\right) \in\{(3,2,1,1),(5,3,2,1)\}$ and from the definition of $c$ and $d$ ) and so in this case $\chi_{\alpha}^{\beta}=(-1)^{c} 2$.

So assume now that $k>4$. As $\left(\alpha_{2}, \ldots, \alpha_{h}\right) \in$ Sign, it follows that $\alpha_{j}>\alpha_{k-1}+\cdots+\alpha_{h}$ for $j \leqslant k-2$. Also

$$
\delta_{2}=\alpha_{3}+\cdots+\alpha_{k-2}+1 \geqslant \alpha_{3}+1>d+2>2 .
$$

So

$$
h_{1, d+2}^{\delta}=\alpha_{2}+d+2-(d+2)=\alpha_{2}
$$

and then as by assumption $|\delta|=\alpha_{2}+\cdots+\alpha_{h}<2 \alpha_{2}$, so that $\delta$ cannot have more than 1 hook of length $\alpha_{2}$,

$$
\chi_{\left(\alpha_{2}, \ldots, \alpha_{h}\right)}^{\delta}=-\chi_{\left(\alpha_{3}, \ldots, \alpha_{h}\right)}^{\epsilon}
$$

with $\epsilon=\left(\alpha_{3}+\cdots+\alpha_{k-2}, d+1,1^{c}\right)$. As $h_{2,1}^{\epsilon}=c+d+1=\alpha_{k-1}+\cdots+\alpha_{h}<\alpha_{j}$ for $j \leqslant k-2$ and then in particular also $\alpha_{k-2} \geqslant d+1>2$, we have that

$$
\chi_{\left(\alpha_{3}, \ldots, \alpha_{h}\right)}^{\epsilon}=\chi_{\left(\alpha_{k-2}, \ldots, \alpha_{h}\right)}^{\left(\alpha_{k-2}, d+1,1^{c}\right)}=-\chi_{\left(\alpha_{k-1}, \ldots, \alpha_{h}\right)}^{\left(d, 1^{c+1}\right)}=(-1)^{c} .
$$

In particular also in this case $\chi_{\alpha}^{\beta}=(-1)^{c} 2$. 
Theorem 3.4. Assume that the following hold:

- $\alpha \notin \operatorname{Sign},\left(\alpha_{2}, \ldots, \alpha_{h}\right) \in \operatorname{Sign}$ and $\alpha_{1}>\alpha_{2}>\alpha_{3}+\cdots+\alpha_{h}$,

- $k \leqslant h$,

- $\alpha_{1}-\alpha_{2}$ is not a part of $\alpha$,

- one of the following holds:

$$
\begin{aligned}
& -\left(\alpha_{k-1}, \ldots, \alpha_{h}\right)=(a, a-1,1) \text { with } a \geqslant 2, \alpha_{1}=\alpha_{2}+\alpha_{k-1}+\cdots+\alpha_{h}-1 \text { and } \\
& \quad\left(\alpha_{k-2}, \ldots, \alpha_{h}\right) \notin\{(3,2,1,1),(5,3,2,1)\}, \\
& -\left(\alpha_{k-1}, \ldots, \alpha_{h}\right)=(a, a-1,2,1) \text { with } a \geqslant 4 \text { and } \alpha_{1}=\alpha_{2}+\alpha_{k-1}+\cdots+\alpha_{h}-3, \\
& -\left(\alpha_{k-1}, \ldots, \alpha_{h}\right)=(a, a-1,3,1) \text { with } a \geqslant 5 \text { and } \alpha_{1}=\alpha_{2}+\alpha_{k-1}+\cdots+\alpha_{h}-4 .
\end{aligned}
$$

Then $\beta:=\left(|\alpha|-\alpha_{1}, 1^{\alpha_{1}}\right)$ is a partition with $h_{2,1}^{\beta}=\alpha_{1}$ and $\chi_{\alpha}^{\beta}=(-1)^{\alpha_{1}-1} 2$.

Proof. From the definition we clearly have that $\beta$ is a partition with $h_{2,1}^{\beta}=\alpha_{1}$.

Notice that from the assumptions $\alpha_{1}=\alpha_{2}+2 a-1$. Also

$$
|\alpha|-\alpha_{1}=\alpha_{2}+\cdots+\alpha_{h}>\alpha_{2}+2 a-1=\alpha_{1}
$$

and so, as $\alpha_{2}>\alpha_{3}+\cdots+\alpha_{h}$, so that any partition of $\alpha_{2}+\cdots+\alpha_{h}$ has at most one hook of length $\alpha_{2}$,

$$
\begin{aligned}
\chi_{\alpha}^{\beta} & =(-1)^{\alpha_{1}-1} \chi_{\left(\alpha_{2}, \ldots, \alpha_{h}\right)}^{\left(|\alpha|-\alpha_{1}\right)}+\chi_{\left(\alpha_{2}, \ldots, \alpha_{h}\right)}^{\left(|\alpha|-2 \alpha_{1}, 1^{\alpha_{1}}\right)} \\
& =(-1)^{\alpha_{1}-1}+(-1)^{\alpha_{2}-1} \chi_{\left(\alpha_{3}, \ldots, \alpha_{h}\right)}^{\left(|\alpha|-2 \alpha_{1}, 1^{\alpha_{1}-\alpha_{2}}\right)} \\
& =(-1)^{\alpha_{1}-1}+(-1)^{\alpha_{1}} \chi_{\left(\alpha_{3}, \ldots, \alpha_{h}\right)}^{\left(|\alpha|-2 \alpha_{1}, 1^{2 a-1}\right)} .
\end{aligned}
$$

Assume first that either $k=4$ or $k>4$ and $\alpha_{k-2} \geqslant 2 a$. Then, as $\alpha_{k-1}+\cdots+\alpha_{h} \geqslant 2 a$ it follows that

$$
\chi_{\left(\alpha_{3}, \ldots, \alpha_{h}\right)}^{\left(|\alpha|-2 \alpha_{1}, 1^{2 a-1}\right)}=\chi_{\left(\alpha_{k-1}, \ldots, \alpha_{h}\right)}^{\left(\alpha_{k-1}+\cdots+\alpha_{h}-2 a+1,1^{2 a-1}\right)}=(-1)^{(a-1)+(a-2)}=-1 .
$$

The second last equality follows from

$$
\left(\alpha_{k-1}+\cdots+\alpha_{h}-2 a+1,1^{2 a-1}\right)= \begin{cases}\left(1^{2 a}\right) & \left(\alpha_{k-1}, \ldots, \alpha_{h}\right)=(a, a-1,1) \\ \left(3,1^{2 a-1}\right) & \left(\alpha_{k-1}, \ldots, \alpha_{h}\right)=(a, a-1,2,1) \\ \left(4,1^{2 a-1}\right) & \left(\alpha_{k-1}, \ldots, \alpha_{h}\right)=(a, a-1,3,1)\end{cases}
$$

so that, by assumption on $a, a-1>h_{1,2}^{\left(\alpha_{k-1}+\cdots+\alpha_{h}-2 a+1,1^{2 a-1}\right)}$ in the last two cases.

Assume now that $k>4$ and $\alpha_{k-2}<2 a \leqslant \alpha_{k-1}+\cdots+\alpha_{h}$. Notice that in this case $\left(\alpha_{k-1}, \ldots, \alpha_{h}\right)=(a, a-1,1)$, as $\left(\alpha_{2}, \ldots, \alpha_{h}\right) \in$ Sign and then also $\left(\alpha_{k-2}, \ldots, \alpha_{h}\right) \in$ Sign. From this assumption and the assumption that $\left(\alpha_{k-2}, \ldots, \alpha_{h}\right) \notin\{(3,2,1,1),(5,3,2,1)\}$ it follows that $\left(\alpha_{k-2}, \ldots, \alpha_{h}\right) \in\{(4,3,2,1),(5,4,3,1)\}$. Also, always by assumption of $\left(\alpha_{2}, \ldots, \alpha_{h}\right) \in$ Sign, if $k \geqslant 6$ then $\alpha_{k-3}>2 a-1$. In either of the two cases

$$
\chi_{\left(\alpha_{3}, \ldots, \alpha_{h}\right)}^{\left(|\alpha|-2 \alpha_{1}, 1^{2 a-1}\right)}=\chi_{\left(\alpha_{k-2}, \ldots, \alpha_{h}\right)}^{\left(\alpha_{k-2}+1,1^{2 a-1}\right)}=-1 .
$$

In either case $\chi_{\alpha}^{\beta}=(-1)^{\alpha_{1}-1} 2$ and so the theorem is proved. 
Theorem 3.5. Assume that the following hold:

- $\alpha \notin \operatorname{Sign},\left(\alpha_{2}, \ldots, \alpha_{h}\right) \in \operatorname{Sign}$ and $\alpha_{1}>\alpha_{2}>\alpha_{3}+\cdots+\alpha_{h}$,

- $k \leqslant h$,

- $\alpha_{1}-\alpha_{2}$ is not a part of $\alpha$,

- $\alpha_{1}=\alpha_{2}+\alpha_{k-1}+\cdots+\alpha_{h}-1$,

- $\left(\alpha_{k-2}, \ldots, \alpha_{h}\right) \in\{(3,2,1,1),(5,3,2,1)\}$.

Then $\beta:=\left(|\alpha|-\alpha_{1}, \alpha_{1}\right)$ is a partition with $h_{2,1}^{\beta}=\alpha_{1}$ and $\chi_{\alpha}^{\beta}=2$.

Proof. Since, by assumption, $\alpha_{1}<\alpha_{2}+\cdots+\alpha_{h}=|\alpha|-\alpha_{1}$ we have that $\beta$ is a partition. Also clearly $h_{2,1}^{\beta}=\alpha_{1}$.

Notice that in this case $k-2>2$, as $\alpha_{k-2}<\alpha_{k-1}+\cdots+\alpha_{h}$ and by assumption $\alpha_{2}>\alpha_{3}+\cdots+\alpha_{h}$. As

$$
1<\alpha_{3}+\cdots+\alpha_{k-2}+3<\alpha_{3}+\cdots+\alpha_{h}<\alpha_{2}<\alpha_{1}
$$

it follows that

$$
\begin{aligned}
h_{1, \alpha_{3}+\cdots+\alpha_{k-2}+3}^{\beta} & =|\alpha|-\alpha_{1}+2-\left(\alpha_{3}+\cdots+\alpha_{k-2}+3\right) \\
& =|\alpha|-\left(\alpha_{2}+\alpha_{k-1}+\cdots+\alpha_{h}-1\right)-\left(\alpha_{3}+\cdots+\alpha_{k-2}\right)-1 \\
& =|\alpha|-\alpha_{2}-\cdots-\alpha_{h} \\
& =\alpha_{1} .
\end{aligned}
$$

So

$$
\chi_{\alpha}^{\beta}=\chi_{\left(\alpha_{2}, \ldots, \alpha_{h}\right)}^{\left(|\alpha|-\alpha_{1}\right)}-\chi_{\left(\alpha_{2}, \ldots, \alpha_{h}\right)}^{\delta}=1-\chi_{\left(\alpha_{2}, \ldots, \alpha_{h}\right)}^{\delta},
$$

with

$$
\delta:=\left(\alpha_{1}-1, \alpha_{3}+\cdots+\alpha_{k-2}+2\right)=\left(\alpha_{2}+\alpha_{k-1}+\cdots+\alpha_{h}-2, \alpha_{3}+\cdots+\alpha_{k-2}+2\right) .
$$

Also by assumption

$$
1<\alpha_{k-1}+\cdots+\alpha_{h}<\alpha_{k-2}+2 \leqslant \alpha_{3}+\cdots+\alpha_{k-2}+2
$$

and then

$$
h_{1, \alpha_{k-1}+\cdots+\alpha_{h}}^{\delta}=\alpha_{2}+\alpha_{k-1}+\cdots+\alpha_{h}-2+2-\alpha_{k-1}+\cdots+\alpha_{h}=\alpha_{2} .
$$

From the previous $\alpha_{3}+\cdots+\alpha_{k-2}+2<\alpha_{2}$ and so

$$
\chi_{\left(\alpha_{2}, \ldots, \alpha_{h}\right)}^{\delta}=-\chi_{\left(\alpha_{3}, \ldots, \alpha_{h}\right)}^{\epsilon}
$$

with

$$
\epsilon:=\left(\alpha_{3}+\cdots+\alpha_{k-2}+1, \alpha_{k-1}+\cdots+\alpha_{h}-1\right) .
$$


As $\left(\alpha_{2}, \ldots, \alpha_{h}\right) \in$ Sign by assumption, so that $\alpha_{j}>\alpha_{k-1}+\cdots+\alpha_{h}>\epsilon_{2}$ for $j \leqslant k-3$ and as $\alpha_{k-2}+1>\alpha_{k-1}+\cdots+\alpha_{h}-1$ by assumption, it follows that

$$
\chi_{\left(\alpha_{3}, \ldots, \alpha_{h}\right)}^{\epsilon}=\chi_{\left(\alpha_{k-2}, \ldots, \alpha_{h}\right)}^{\left(\alpha_{k-2}+1, \alpha_{k-1}+\cdots+\alpha_{h}-1\right)}=1
$$

(the last equation follows from the assumption that $\left(\alpha_{k-2}, \ldots, \alpha_{h}\right)$ is either $(3,2,1,1)$ or $(5,3,2,1))$.

In particular $\chi_{\alpha}^{\beta}=2$ and so the theorem holds.

Theorem 3.6. Assume that the following hold:

- $\alpha \notin \operatorname{Sign},\left(\alpha_{2}, \ldots, \alpha_{h}\right) \in \operatorname{Sign}$ and $\alpha_{1}>\alpha_{2}>\alpha_{3}+\cdots+\alpha_{h}$,

- $k \leqslant h$,

- there exists $i$ with $\alpha_{i}=\alpha_{1}-\alpha_{2}$,

- $\alpha_{i} \geqslant \alpha_{i+1}+\cdots+\alpha_{h}$.

Then $\beta=\left(|\alpha|-\alpha_{1}, \alpha_{2}+1,1^{\alpha_{1}-\alpha_{2}-1}\right)$ is a partition with $h_{2,1}^{\beta}=\alpha_{1}$ and $\chi_{\alpha}^{\beta}=(-1)^{\alpha_{1}-\alpha_{2}-1} 2$.

Proof. Since by assumption $\alpha_{1}>\alpha_{2}+\alpha_{h} \geqslant \alpha_{2}+1$ and (also using Lemma 1.5)

$$
|\alpha|-\alpha_{1} \geqslant \alpha_{1}>\alpha_{2}+\alpha_{h} \geqslant \alpha_{2}+1
$$

it follows that $\beta$ is partition. Also clearly $h_{2,1}^{\beta}=\alpha_{1}$.

From the definition of $k$ and from

$$
2 \alpha_{2}>\alpha_{2}+\cdots+\alpha_{h} \geqslant \alpha_{1}
$$

we have that $3 \leqslant i<k \leqslant h$. Then

$$
\begin{aligned}
h_{1,2}^{\beta} & =|\alpha|-\alpha_{1}=\alpha_{2}+\cdots+\alpha_{h} \geqslant \alpha_{2}+\alpha_{i}+\alpha_{h}>\alpha_{1}, \\
h_{1, \alpha_{2}+1}^{\beta} & =|\alpha|-\alpha_{1}+2-\alpha_{2}-1=\alpha_{3}+\cdots+\alpha_{h}+1 \leqslant \alpha_{2}<\alpha_{1} .
\end{aligned}
$$

In particular there exists $3 \leqslant j \leqslant \alpha_{2}$ such that $h_{1, j}^{\beta}=\alpha_{1}$. From the Murnaghan-Nakayama formula it follows that

$$
\begin{aligned}
\chi_{\alpha}^{\beta} & =(-1)^{\alpha_{1}-\alpha_{2}-1} \chi_{\left(\alpha_{2}, \ldots, \alpha_{h}\right)}^{\left(|\alpha|-\alpha_{1}\right)}-\chi_{\left(\alpha_{2}, \ldots, \alpha_{3}\right)}^{\left(\alpha_{2}, j-1,1^{\alpha_{1}-\alpha_{2}-1}\right)} \\
& =(-1)^{\alpha_{1}-\alpha_{2}-1}+\chi_{\left(\alpha_{3}, \ldots, \alpha_{h}\right)}^{\left(j-2, \alpha_{1}-\alpha_{2}\right)} \\
& =(-1)^{\alpha_{1}-\alpha_{2}-1}+\chi_{\left(\alpha_{i}, \ldots, \alpha_{h}\right)}^{\left(\alpha_{i+1}+\cdots+\alpha_{h}, 1^{\alpha_{i}}\right)} \\
& =(-1)^{\alpha_{1}-\alpha_{2}-1}+(-1)^{\alpha_{i}-1} \chi_{\left(\alpha_{i+1}, \ldots, \alpha_{h}\right)}^{\left(\alpha_{i+1}+\cdots+\alpha_{h}\right)} \\
& =(-1)^{\alpha_{1}-\alpha_{2}-1} 2 .
\end{aligned}
$$

The second line follows from $h_{1,2}^{\left(\alpha_{2}, j-1,1^{\alpha_{1}-\alpha_{2}-1}\right)}=\alpha_{2}$, as $j \geqslant 3$, and from

$$
\left|\left(\alpha_{2}, j-1,1^{\alpha_{1}-\alpha_{2}-1}\right)\right|=|\alpha|-\alpha_{1}<2 \alpha_{2},
$$


so that $\left(\alpha_{2}, j-1,1^{\alpha_{1}-\alpha_{2}-1}\right)$ has at most one hook of length $\alpha_{2}$. The third line from $\alpha_{j}>\alpha_{i}$ for $j<i$ and from $i<h$, so that

$$
\begin{aligned}
h_{1,2}^{\left(j-2,1^{\alpha_{1}-\alpha_{2}}\right)} & =\left|\left(\alpha_{3}, \ldots, \alpha_{h}\right)\right|-\left(\alpha_{1}-\alpha_{2}\right)-1 \\
& =\alpha_{3}+\cdots+\alpha_{h}-\alpha_{i}-1 \\
& \geqslant \alpha_{1}+\cdots+\alpha_{i+1} .
\end{aligned}
$$

The fourth line follows from $\alpha_{i} \geqslant \alpha_{i+1}+\cdots+\alpha_{h}$.

Theorem 3.7. Assume that the following hold:

- $\alpha \notin \operatorname{Sign},\left(\alpha_{2}, \ldots, \alpha_{h}\right) \in \operatorname{Sign}$ and $\alpha_{1}>\alpha_{2}>\alpha_{3}+\cdots+\alpha_{h}$,

- $k \leqslant h$,

- there exists $i$ with $\alpha_{i}=\alpha_{1}-\alpha_{2}$,

- $\alpha_{i}<\alpha_{i+1}+\cdots+\alpha_{h}$.

Then $\beta=\left(|\alpha|-\alpha_{1}, \alpha_{2}+2,1^{\alpha_{1}-\alpha_{2}-2}\right)$ is a partition with $h_{2,1}^{\beta}=\alpha_{1}$ and $\chi_{\alpha}^{\beta}=(-1)^{\alpha_{1}-\alpha_{2}} 2$.

Proof. Since by assumption $\alpha_{1}>\alpha_{2}+\alpha_{h} \geqslant \alpha_{2}+1$ and

$$
|\alpha|-\alpha_{1} \geqslant \alpha_{1}>\alpha_{2}+\alpha_{h} \geqslant \alpha_{2}+1
$$

it follows that $\beta$ is partition with $h_{2,1}^{\beta}=\alpha_{1}$.

From $\alpha_{i}<\alpha_{i+1}+\cdots+\alpha_{h}$ and $\left(\alpha_{2}, \ldots, \alpha_{h}\right) \in$ Sign it follows that

$$
\begin{gathered}
\left(\alpha_{i}, \ldots, \alpha_{h}\right) \in\{(3,2,1,1),(5,3,2,1)\} \cup\{(a, a-1,2,1): a \geqslant 4\} \\
\cup\{(a, a-1,3,1): a \geqslant 5\} .
\end{gathered}
$$

Similar to the previous theorem we have that $3 \leqslant i<k \leqslant h$, from which follows that

$$
\begin{aligned}
h_{1,2}^{\beta} & =|\alpha|-\alpha_{1}=\alpha_{2}+\cdots+\alpha_{h} \geqslant \alpha_{2}+\alpha_{i}+\cdots+\alpha_{h} \geqslant \alpha_{1}+2, \\
h_{1, \alpha_{2}+2}^{\beta} & =|\alpha|-\alpha_{1}+2-\alpha_{2}-2=\alpha_{3}+\cdots+\alpha_{h}<\alpha_{2}<\alpha_{1} .
\end{aligned}
$$

In particular there exists $4 \leqslant j \leqslant \alpha_{2}$ such that $h_{1, j}^{\beta}=\alpha_{1}$. So

$$
\begin{aligned}
\chi_{\alpha}^{\beta} & =(-1)^{\alpha_{1}-\alpha_{2}-2} \chi_{\left(\alpha_{2}, \ldots, \alpha_{h}\right)}^{\left(|\alpha|-\alpha_{1}\right)}-\chi_{\left(\alpha_{2}, \ldots, \alpha_{3}\right)}^{\left(\alpha_{2}+1, j-1,1^{\alpha_{1}-\alpha_{2}-2}\right)} \\
& \left.=(-1)^{\alpha_{1}-\alpha_{2}}+\chi_{\left(\alpha_{3}, \ldots, \alpha_{h}\right)}^{\left(j-2,2, \alpha_{1}-\alpha_{2}-2\right.}\right) \\
& =(-1)^{\alpha_{1}-\alpha_{2}}+\chi_{\left(\alpha_{i}, \ldots, \alpha_{h}\right)}^{\left(\alpha_{i+1}+\cdots+\alpha_{h}, 2,1^{\alpha_{i}-2}\right)}
\end{aligned}
$$

The second line follows from $\alpha_{2}>\alpha_{3}+\cdots+\alpha_{h}$ and, as $j \geqslant 4$,

$$
h_{1,3}^{\left(\alpha_{2}+1, j-1,1^{\alpha_{1}-\alpha_{2}-2}\right)}=\alpha_{2}+1+2-3=\alpha_{2} .
$$


The third line follows from $\alpha_{j}>\alpha_{i}$ for $j<i$ and from

$$
\begin{aligned}
h_{1,3}^{\left(j-2,2,1^{\alpha_{1}-\alpha_{2}-2}\right)} & =\left|\left(\alpha_{3}, \ldots, \alpha_{h}\right)\right|-\left(\alpha_{1}-\alpha_{2}\right)-2 \\
& =\alpha_{3}+\cdots+\alpha_{h}-\alpha_{i}-2 \\
& \geqslant \alpha_{1}+\cdots+\alpha_{i+1} .
\end{aligned}
$$

If $\left(\alpha_{i}, \ldots, \alpha_{h}\right) \in\{(3,2,1,1),(5,3,2,1)\}$ it is easy to check that

$$
\chi_{\left(\alpha_{i}, \ldots, \alpha_{h}\right)}^{\left(\alpha_{i+1}+\cdots+\alpha_{h}, 2,1^{\alpha_{i}-2}\right)}=-1=(-1)^{\alpha_{i}}=(-1)^{\alpha_{1}-\alpha_{2}} .
$$

In particular the theorem holds in this case.

If $\left(\alpha_{i}, \ldots, \alpha_{h}\right)=(a, a-1, c, 1)$ with $c \in\{2,3\}$ then, as $a-1>c$,

$$
\begin{aligned}
\chi_{\left(\alpha_{i}, \ldots, \alpha_{h}\right)}^{\left(\alpha_{i+1}+\cdots+\alpha_{h}, 2,1^{\alpha_{i}-2}\right)} & =\chi_{(a, a-1, c, 1)}^{\left(a+c, 2,1^{a-2}\right)} \\
& =(-1)^{a-2} \chi_{(a-1, c, 1)}^{(a+c)}+\chi^{\left(c, 2,1^{a-2}\right)} \\
& =(-1)^{a} \\
& =(-1)^{\alpha_{1}-\alpha_{2}},
\end{aligned}
$$

so that the theorem holds also in this case.

In the next theorems we will consider the case $k=h+1$, that is $\alpha_{1}-\alpha_{2} \leqslant \alpha_{h}$.

Theorem 3.8. Assume that the following hold:

- $\alpha \notin \operatorname{Sign},\left(\alpha_{2}, \ldots, \alpha_{h}\right) \in \operatorname{Sign}$ and $\alpha_{1}>\alpha_{2}>\alpha_{3}+\cdots+\alpha_{h}$,

- $\alpha_{1}-\alpha_{2}<\alpha_{h}$.

Then $\beta:=\left(|\alpha|-\alpha_{1}, 1^{\alpha_{1}}\right)$ is a partition with $h_{2,1}^{\beta}=\alpha_{1}$ and $\chi_{\alpha}^{\beta}=(-1)^{\alpha_{1}-1} 2$.

Proof. Clearly $\beta$ is a partition and $h_{2,1}^{\beta}=\alpha_{1}$. By assumption $|\alpha|-\alpha_{1} \geqslant \alpha_{2}+\alpha_{h}>\alpha_{1}$, from which also follows that $\alpha_{1}-\alpha_{2}<\alpha_{h} \leqslant \alpha_{j}$ for $j \leqslant h$. Also as by assumption $\alpha_{2}>\alpha_{3}+\cdots+\alpha_{h}$, so that any partition of $\alpha_{2}+\cdots+\alpha_{h}$ has at most one $\alpha_{2}$-hook, it follows from the Murnaghan-Nakayama formula that

$$
\begin{aligned}
\chi_{\alpha}^{\beta} & =(-1)^{\alpha_{1}-1} \chi_{\left(\alpha_{2}, \ldots, \alpha_{h}\right)}^{\left(|\alpha|-\alpha_{1}\right)}+\chi_{\left(\alpha_{2}, \ldots, \alpha_{h}\right)}^{\left(|\alpha|-2 \alpha_{1}, 1^{\alpha_{1}}\right)} \\
& =(-1)^{\alpha_{1}-1}+(-1)^{\alpha_{2}-1} \chi_{\left(\alpha_{3}, \ldots, \alpha_{h}\right)}^{\left(|\alpha|-2 \alpha_{1}, 1^{\left.\alpha_{1}-\alpha_{2}\right)}\right.} \\
& =(-1)^{\alpha_{1}-1}+(-1)^{\alpha_{2}-1} \chi_{\left(\alpha_{h}\right)}^{\left(\alpha_{h}-\alpha_{1}+\alpha_{2}, 1^{\alpha_{1}-\alpha_{2}}\right)} \\
& =(-1)^{\alpha_{1}-1} 2 .
\end{aligned}
$$

Theorem 3.9. Assume that the following hold:

- $\alpha \notin \operatorname{Sign},\left(\alpha_{2}, \ldots, \alpha_{h}\right) \in \operatorname{Sign}$ and $\alpha_{1}>\alpha_{2}>\alpha_{3}+\cdots+\alpha_{h}$, 
- $\alpha_{1}-\alpha_{2}=\alpha_{h}$,

- $h=3$.

Then $\beta=\left(\alpha_{1}, \alpha_{1}\right)$ is a partitions with $h_{2,1}^{\beta}=\alpha_{1}$ and $\chi_{\alpha}^{\beta}=2$.

Proof. Notice that $\alpha_{3} \geqslant 2$, since $1 \leqslant \alpha_{1}-\alpha_{2}=\alpha_{3}$ and $\left(\alpha_{1}, \alpha_{2}, \alpha_{3}\right) \notin$ Sign. Clearly $\beta$ is a partition with $h_{2,1}^{\beta}=\alpha_{1}$.

As $\beta=\left(\alpha_{1}, \alpha_{1}\right)$ and $\alpha_{3} \geqslant 2$ we have that

$$
\chi_{\alpha}^{\beta}=\chi_{\left(\alpha_{2}, \alpha_{3}\right)}^{\left(\alpha_{1}\right)}-\chi_{\left(\alpha_{2}, \alpha_{3}\right)}^{\left(\alpha_{1}-1,1\right)}=2 .
$$

Theorem 3.10. Assume that the following hold:

- $\alpha \notin \operatorname{Sign},\left(\alpha_{2}, \ldots, \alpha_{h}\right) \in \operatorname{Sign}$ and $\alpha_{1}>\alpha_{2}>\alpha_{3}+\cdots+\alpha_{h}$,

- $\alpha_{1}-\alpha_{2}=\alpha_{h} \geqslant 2$,

- $h \geqslant 4$.

Then $\beta=\left(|\alpha|-\alpha_{1}, \alpha_{2}+2,1^{\alpha_{1}-\alpha_{2}-2}\right)$ is a partition with $h_{2,1}^{\beta}=\alpha_{1}$ and $\chi_{\alpha}^{\beta}=(-1)^{\alpha_{1}-\alpha_{2}} 2$.

Proof. As $\alpha_{2}+2 \leqslant \alpha_{2}+\alpha_{h}=\alpha_{1}$ and $|\alpha|-\alpha_{1} \geqslant \alpha_{2}+\alpha_{h}$ we have that $\beta$ is a partition and that $h_{2,1}^{\beta}=\alpha_{1}$. Notice that $\beta_{1}^{\prime}$, which is the number of parts of $\beta$, is given by

$$
\beta_{1}^{\prime}=\alpha_{1}-\alpha_{2}=\alpha_{h}
$$

As $h \geqslant 4$ and $\alpha_{h-1}>\alpha_{h} \geqslant 2$ we have that

$$
\begin{aligned}
h_{1,2}^{\beta} & =|\alpha|-\alpha_{1} \geqslant \alpha_{2}+\alpha_{h}+\alpha_{h-1} \geqslant \alpha_{1}+3, \\
h_{1, \alpha_{2}+2}^{\beta} & =|\alpha|-\alpha_{1}-\alpha_{2}=\alpha_{3}+\cdots+\alpha_{h} \leqslant \alpha_{2}-1 \leqslant \alpha_{1}-2 .
\end{aligned}
$$

In particular there exists $5 \leqslant j \leqslant \alpha_{2}$ with $h_{1, j}^{\beta}=\alpha_{1}$. Such $j$ satisfies $\beta \backslash R_{1, j}^{\beta}=\left(\alpha_{2}+\right.$ $1, j-1,1^{\alpha_{1}-\alpha_{2}-2}$ ) and then also $h_{1,3}^{\beta \backslash R_{1, j}^{\beta}}=\alpha_{2}$ as $j-1>3$ (where $R_{1, j}^{\beta}$ is the rim hook of $\beta$ corresponding to node $(1, j))$. As $\alpha_{2}>\alpha_{3}+\cdots+\alpha_{h}$, as $\beta_{1}^{\prime}=\alpha_{h}$ and as $\alpha_{i}>\alpha_{h}$ for $i<h$ (since $\alpha_{h} \geqslant 2$ ) we then obtain from the Murnaghan-Nakayama formula that

$$
\begin{aligned}
\chi_{\alpha}^{\beta} & =(-1)^{\alpha_{1}-\alpha_{2}-2} \chi_{\left(\alpha_{2}, \ldots, \alpha_{h}\right)}^{\left(|\alpha|-\alpha_{1}\right)}-\chi_{\left(\alpha_{2}, \ldots, \alpha_{h}\right)}^{\left(\alpha_{2}+1, j-1,1^{\alpha_{1}-\alpha_{2}-2}\right)} \\
& =(-1)^{\alpha_{1}-\alpha_{2}}+\chi_{\left(\alpha_{3}, \ldots, \alpha_{h}\right)}^{\left(j-2, \alpha_{h}\right)} \\
& \left.=(-1)^{\alpha_{1}-\alpha_{2}}+\chi_{\left(\alpha_{h-1}, \alpha_{h}\right)}^{\left(\alpha_{h-1}, 1_{h}-2\right.}\right) \\
& =(-1)^{\alpha_{1}-\alpha_{2}}-\chi_{\left(\alpha_{h-1}, \alpha_{h}\right)}^{\left(1^{\alpha_{h}}\right)} \\
& =(-1)^{\alpha_{1}-\alpha_{2}}+(-1)^{\alpha_{h}} \\
& =(-1)^{\alpha_{1}-\alpha_{2}} 2 .
\end{aligned}
$$


Theorem 3.11. Assume that the following hold:

- $\alpha \notin \operatorname{Sign},\left(\alpha_{2}, \ldots, \alpha_{h}\right) \in \operatorname{Sign}$ and $\alpha_{1}>\alpha_{2}>\alpha_{3}+\cdots+\alpha_{h}$,

- $\alpha_{1}-\alpha_{2}=\alpha_{h}=1=\alpha_{h-1}$,

- $h \geqslant 4$.

Then $\beta=\left(|\alpha|-\alpha_{1}, \alpha_{1}\right)$ is a partition with $h_{2,1}^{\beta}=\alpha_{1}$ and $\chi_{\alpha}^{\beta}=2$.

Proof. From Lemma 1.5 it follows from the assumptions that $|\alpha|-\alpha_{1} \geqslant \alpha_{1}$ and so $\beta$ is a partition. Also $h_{2,1}^{\beta}=\alpha_{1}$. As

$$
3=\alpha_{h-1}+2 \leqslant \alpha_{3}+\cdots+\alpha_{h-1}+2=\alpha_{3}+\cdots+\alpha_{h}+1 \leqslant \alpha_{2}<\alpha_{1}
$$

and

$$
|\alpha|-2 \alpha_{1}+2=\alpha_{2}+\cdots+\alpha_{h}-\alpha_{1}+2=\alpha_{3}+\cdots+\alpha_{h-1}+2,
$$

we have that, for $j=|\alpha|-2 \alpha_{1}+2$,

$$
h_{1, j}^{\beta}=|\alpha|-\alpha_{1}+2-j=\alpha_{1} .
$$

Also $2 \leqslant j-1<\alpha_{2}$ and then, as $\alpha_{2}=\alpha_{1}-1$ and $\alpha_{h-2}>\alpha_{h-1}=\alpha_{h}=1$,

$$
\chi_{\alpha}^{\beta}=\chi_{\left(\alpha_{2} \ldots, \alpha_{h}\right)}^{\left(|\alpha|-\alpha_{1}\right)}-\chi_{\left(\alpha_{2}, \ldots, \alpha_{h}\right)}^{\left(\alpha_{1}-1, j-1\right)}=1+\chi_{\left(\alpha_{3}, \ldots, \alpha_{h}\right)}^{(j-2,1)}=2 .
$$

Theorem 3.12. Assume that the following hold:

- $\alpha \notin \operatorname{Sign},\left(\alpha_{2}, \ldots, \alpha_{h}\right) \in \operatorname{Sign}$ and $\alpha_{1}>\alpha_{2}>\alpha_{3}+\cdots+\alpha_{h}$,

- $\alpha_{1}-\alpha_{2}=\alpha_{h}=1<\alpha_{h-1}$,

- $h=4$.

Then $\beta=\left(\alpha_{1}-2, \alpha_{3}, \alpha_{3}, 4,1^{\alpha_{1}-\alpha_{3}-2}\right)$ is a partition with $h_{2,1}^{\beta}=\alpha_{1}$ and $\chi_{\alpha}^{\beta}=(-1)^{\alpha_{1}-\alpha_{3}} 2$.

Proof. Notice that from the assumptions it follows that $\alpha_{3} \geqslant 4$. Also $\alpha_{1}>\alpha_{2}>\alpha_{3}$ and so $\beta$ is a partition with $h_{2,1}^{\beta}=\alpha_{1}$. As $\alpha_{2}=\alpha_{1}-1$ and $\alpha_{4}=1$ we have that

$$
\begin{aligned}
\chi_{\alpha}^{\beta} & =(-1)^{\alpha_{1}-\alpha_{3}} \chi_{\left(\alpha_{1}-1, \alpha_{3}, 1\right)}^{\left(\alpha_{1}-2, \alpha_{3}-1,3\right)}-\chi_{\left(\alpha_{1}-1, \alpha_{3}, 1\right)}^{\left(\alpha_{3}-1, \alpha_{3}-1,3,1^{\alpha_{1}-\alpha_{3}-1}\right)} \\
& =(-1)^{\alpha_{1}-\alpha_{3}} \chi_{\left(\alpha_{3}, 1\right)}^{\left(\alpha_{3}-2,1\right)}+(-1)^{\alpha_{1}-\alpha_{3}+1} \chi_{\left(\alpha_{3}, 1\right)}^{\left(\alpha_{3}-1,2\right)} \\
& =(-1)^{\alpha_{1}-\alpha_{3}} 2 .
\end{aligned}
$$

Theorem 3.13. Assume that the following hold:

- $\alpha \notin \operatorname{Sign},\left(\alpha_{2}, \ldots, \alpha_{h}\right) \in \operatorname{Sign}$ and $\alpha_{1}>\alpha_{2}>\alpha_{3}+\cdots+\alpha_{h}$, 
- $\alpha_{1}-\alpha_{2}=\alpha_{h}=1$,

- $h \geqslant 5$,

- $\alpha_{h-1}=2$.

Then $\beta=\left(|\alpha|-\alpha_{1}-2, \alpha_{1}-2,2,2\right)$ is a partition with $h_{2,1}^{\beta}=\alpha_{1}$ and $\chi_{\alpha}^{\beta}=-2$.

Proof. As $\alpha_{1}>\alpha_{2}>\ldots>\alpha_{h}=1$ it follows that $\alpha_{1} \geqslant h \geqslant 5$. Also, by assumption on $\alpha$,

$$
|\alpha|-\alpha_{1} \geqslant \alpha_{2}+\alpha_{h-2}+\alpha_{h} \geqslant \alpha_{1}+3
$$

and so it follows that $\beta$ is a partition. Clearly $h_{2,1}^{\beta}=\alpha_{1}$. Since by assumption

$$
|\alpha|-2 \alpha_{1}+2=\alpha_{2}+\cdots+\alpha_{h}-\alpha_{1}+2=\alpha_{3}+\cdots+\alpha_{h}+1 \leqslant \alpha_{2}<\alpha_{1}
$$

we also have that

$$
\begin{aligned}
h_{1,3}^{\beta} & =|\alpha|-\alpha_{1}-2+2-3=|\alpha|-\alpha_{1}-3 \geqslant \alpha_{1}, \\
h_{1, \alpha_{1}-2}^{\beta} & =|\alpha|-\alpha_{1}-2+2-\alpha_{1}+2=|\alpha|-2 \alpha_{1}+2<\alpha_{1} .
\end{aligned}
$$

In particular there exists $3 \leqslant j \leqslant \alpha_{1}-3$ with $h_{1, j}^{\beta}=\alpha_{1}$.

From $\alpha_{h-1}=2$ and $\alpha_{h}=1$ it follows that $\alpha_{j}+\cdots+\alpha_{h}-3 \geqslant \alpha_{j}$ for $j \leqslant h-2$. Since $\alpha_{j} \geqslant 3$ for $j \leqslant h-2$ we then have that

$$
\begin{aligned}
\chi_{\alpha}^{\beta} & =\chi_{\left(\alpha_{2}, \ldots, \alpha_{h}\right)}^{\left(|\alpha|-\alpha_{1}-2,1,1\right)}-\chi_{\left(\alpha_{2}, \ldots, \alpha_{h}\right)}^{\left(\alpha_{1}-3, j-1,2,2\right)} \\
& =\chi_{\left(\alpha_{3}, \ldots, \alpha_{h}\right)}^{\left(|\alpha|-\alpha_{1}-\alpha_{2}-2,1,1\right)}+\chi_{\left(\alpha_{3}, \ldots, \alpha_{h}\right)}^{(j-2,1,1)} \\
& =2 \chi_{(2,1)}^{(1,1,1)} \\
& =-2 .
\end{aligned}
$$

Theorem 3.14. Assume that the following hold:

- $\alpha \notin \operatorname{Sign},\left(\alpha_{2}, \ldots, \alpha_{h}\right) \in \operatorname{Sign}$ and $\alpha_{1}>\alpha_{2}>\alpha_{3}+\cdots+\alpha_{h}$,

- $\alpha_{1}-\alpha_{2}=\alpha_{h}=1$,

- $h \geqslant 5$,

- $\alpha_{h-1} \geqslant 3$.

Then $\beta=\left(|\alpha|-\alpha_{1}-\alpha_{h-1}+1,3,3,2^{\alpha_{h-1}-3}, 1^{\alpha_{1}-\alpha_{h-1}-1}\right)$ is a partition with $h_{2,1}^{\beta}=\alpha_{1}$ and $\chi_{\alpha}^{\beta}=(-1)^{\alpha_{1}+\alpha_{h-1}-1} 2$. 
Proof. As $h \geqslant 5$, so that

$$
\beta_{1}=|\alpha|-\alpha_{1}-\alpha_{h-1}+1 \geqslant \alpha_{2}+\alpha_{3}+1>\alpha_{1}+3,
$$

and as $\alpha_{1}>\alpha_{h-1} \geqslant 3$ it follows that $\beta$ is a partition with $h_{2,1}^{\beta}=\alpha_{1}$. Also $\beta_{1} \geqslant 4$ and $h_{1,4}^{\beta} \geqslant \alpha_{1}$. From the assumptions we also have

$$
|\alpha|-2 \alpha_{1}-\alpha_{h-1}=\alpha_{2}+\cdots+\alpha_{h}-\alpha_{1}-\alpha_{h-1}=\alpha_{3}+\cdots+\alpha_{h-2}>\alpha_{3}+\cdots+\alpha_{h-3}+2 .
$$

Since $\alpha_{j}>\alpha_{h-1}$ for $j<h-1$ and again any partition of $\alpha_{2}+\cdots+\alpha_{h}$ has at most one $\alpha_{2}$-hook, we have that

$$
\begin{aligned}
\chi_{\alpha}^{\beta} & =(-1)^{\alpha_{1}-3} \chi_{\left(\alpha_{2}, \ldots, \alpha_{h}\right)}^{\left(|\alpha|-\alpha_{h-1}+1,2,1^{\alpha_{h-1}-3}\right)}+\chi_{\left(\alpha_{2}, \ldots, \alpha_{h}\right)}^{\left(|\alpha|-2 \alpha_{1}-\alpha_{h-1}+1,3,3,2^{\alpha_{h-1}-3}, 1^{\alpha_{1}-\alpha_{h-1}-1}\right)} \\
& =(-1)^{\alpha_{1}-1} \chi_{\left(\alpha_{3}, \ldots, \alpha_{h}\right)}^{\left(|\alpha|-2 \alpha_{1}-\alpha_{h-1}+2,2,1^{\alpha_{h-1}-3}\right)}+(-1)^{\alpha_{1}-4} \chi_{\left(\alpha_{3}, \ldots, \alpha_{h}\right)}^{\left(|\alpha|-2 \alpha_{1}-\alpha_{h-1}+1,3,1^{\alpha_{h-1}-3}\right)} \\
& \left.\left.=(-1)^{\alpha_{1}-1} \chi_{\left(\alpha_{h-2}, \alpha_{h-1}, \alpha_{h}\right)}^{\left(\alpha_{h-2}+2, \alpha_{h-1}-3\right.}\right)+(-1)^{\alpha_{1}} \chi_{\left(\alpha_{h-2}, \alpha_{h-1}, \alpha_{h}\right)}^{\left(\alpha_{h-2}+1,3, \alpha_{h-1}-3\right.}\right) \\
& =(-1)^{\alpha_{1}-1} 2 \chi_{\left(\alpha_{h-1}, \alpha_{h}\right)}^{\left(2,2, \alpha_{h-1} \alpha_{h-3}\right)} \\
& =(-1)^{\alpha_{1}+\alpha_{h-1}-1} 2 .
\end{aligned}
$$

\section{The partitions $\left(\gamma_{s+1}, \ldots, \gamma_{r}\right)$ are sign partitions}

In this section we will prove that

- ()$,(1,1),(3,2,1,1),(5,3,2,1)$,

- $(a, a-1,1)$ with $a \geqslant 2$,

- $(a, a-1,2,1)$ with $a \geqslant 4$,

- $(a, a-1,3,1)$ with $a \geqslant 5$

are all sign partitions. For ()$,(1,1),(3,2,1,1)$ and $(5,3,2,1)$ this can be done by just looking at the corresponding character table. For the other partitions we will use the next lemma.

Lemma 4.1. Let $a \geqslant 2$ and $\gamma=\left(a, a-1, \gamma_{3}, \ldots, \gamma_{r}\right)$ be a partition. Assume that the following hold.

- $\left(a-1, \gamma_{3}, \ldots, \gamma_{r}\right)$ is a sign partition,

- $\gamma_{3}+\cdots+\gamma_{r} \leqslant a$.

If $\beta$ is a partition of $|\gamma|$ for which $\chi_{\gamma}^{\beta} \notin\{0, \pm 1\}$ then $\beta$ has two a-hooks. Also if $\delta$ is obtained from $\beta$ by removing an a-hook then $\chi_{\left(a-1, \gamma_{3}, \ldots, \gamma_{r}\right)}^{\delta} \neq 0$. In particular each such $\delta$ has an $(a-1)$-hook. 
Proof. By assumption

$$
|\gamma|=2 a-1+\gamma_{3}+\cdots+\gamma_{r}<3 a .
$$

In particular any partition of $|\gamma|$ has at most two $a$-hooks. As

$$
\chi_{\gamma}^{\beta}=\sum_{(i, j): h_{i, j}^{\beta}=a} \pm \chi_{\left(a-1, \gamma_{3}, \ldots, \gamma_{r}\right)}^{\beta \backslash R_{i, j}^{\beta}}
$$

and, since $\left(a-1, \gamma_{3}, \ldots, \gamma_{r}\right)$ is a sign partition, so that $\chi_{\left(a-1, \gamma_{3}, \ldots, \gamma_{r}\right)}^{\beta \backslash R_{i, j}^{\beta}} \in\{0, \pm 1\}$ for each $(i, j) \in[\beta]$, the Young diagram of $\beta$, with $h_{i, j}^{\beta}=a$, the lemma follows.

Theorem 4.2. If $a \geqslant 2$ then $(a, a-1,1)$ is a sign partition.

Proof. As $(a-1,1)$ is a sign partition for $a \geqslant 2$, from Lemma 4.1 we only need to check that $\chi_{(a, a-1,1)}^{\beta} \in\{0, \pm 1\}$ for partitions $\beta$ of $2 a$ with two $a$-hooks and such that if $\mu$ and $\nu$ are the partitions obtained from $\beta$ by removing an $a$-hook then $\mu$ and $\nu$ both have an an $(a-1)$-hook. From $\beta$ having two $a$-hooks it follows that $\mu$ and $\nu$ also have an $a$-hook. The only partitions of $a$ having both an $a$-hook and an $(a-1)$-hook are $(a)$ and $\left(1^{a}\right)$. As $\mu \neq \nu$ it then follows that $\{\mu, \nu\}=\left\{(a),\left(1^{a}\right)\right\}$. Looking at the $a$-quotients and $a$-cores of $\beta, \mu$ and $\nu$ we have that there exists a unique such $\beta$, which is given by $\beta=\left(a, 2,1^{a-2}\right)$. We have

$$
\chi_{(a, a-1,1)}^{\left(a, 2,1^{a-2}\right)}=(-1)^{a-2} \chi_{(a-1,1)}^{(a)}-\chi_{(a-1,1)}^{\left(1^{a}\right)}=(-1)^{a}+(-1)^{a-1}=0
$$

and so $(a, a-1,1)$ is a sign partition.

Theorem 4.3. If $a \geqslant 4$ then $(a, a-1,2,1)$ is a sign partition.

Proof. For $a=4$ we can check that $(a, a-1,2,1)=(4,3,2,1)$ is a sign partition by looking at the character table of $S_{10}$. So assume that $a \geqslant 5$. As $(a-1,2,1)$ is a sign partition for $a \geqslant 5$ from Lemma 1.5, from Lemma 4.1 we only need to check that $\chi_{(a, a-1,2,1)}^{\beta} \in\{0, \pm 1\}$ for partitions $\beta$ of $2 a+2$ with two $a$-hooks and such that if $\mu$ and $\nu$ are the partitions obtained from $\beta$ by removing an $a$-hook then $\mu$ and $\nu$ have both an $a$-hook and an $(a-1)$ hook.

So let $\beta$ have two $a$-hook. Then, as $|\beta|=2 a+2<3 a$, we have that $\beta_{(a)}$, the $a$-core of $\beta$, is either $(2)$ or $\left(1^{2}\right)$. We will assume that $\beta_{(a)}=(2)$, since for any partitions $\lambda, \rho$ with $|\lambda|=|\rho|$ and any positive integer $q$, we have that $\chi_{\rho}^{\lambda}= \pm \chi_{\rho}^{\lambda^{\prime}}$ and $\lambda_{(q)}^{\prime}=\left(\lambda_{(q)}\right)^{\prime}$, where $\lambda^{\prime}$ is the adjoint partition of $\lambda$ and similarly for $\lambda_{(q)}$. Then $\mu$ and $\nu$ can be obtained by adding an $a$-hook to (2) and so

$$
\mu, \nu \in\left\{(a+2),\left(2,2,1^{a-2}\right),\left(2,1^{a}\right)\right\} \cup\left\{\left(a-i, 3,1^{i-1}\right): 1 \leqslant i \leqslant a-3\right\},
$$

as all these partitions can be obtained by adding an $a$-hook to (2) and, since $2<a$, there are exactly $a$ such partitions. As $\mu$ and $\nu$ have an $(a-1)$-hook we then have that

$$
\mu, \nu \in\left\{(a+2),\left(2,1^{a}\right),(a-1,3),\left(3,3,1^{a-4}\right)\right\} .
$$


Notice that since $a \geqslant 5$ the four above partitions are distinct. As $a \geqslant 5$

$$
\begin{aligned}
& \chi_{(a-1,2,1)}^{\left(2,1^{a}\right)}=(-1)^{a-2} \chi_{(2,1)}^{(2,1)}=0, \\
& \chi_{(a-1,2,1)}^{(a-1,3)}=-\chi_{(2,1)}^{(2,1)}=0,
\end{aligned}
$$

we only need to consider, from Lemma 4.1, the partition $\beta$ corresponding to $\{\mu, \nu\}=$ $\left\{(a+2),\left(3,3,1^{a-4}\right)\right\}$, that is for $\beta=\left(a+2,4,1^{a-4}\right)$. As

$$
\chi_{(a, a-1,2,1)}^{\left(a+2,4,1^{a-4}\right)}=-\chi_{(a-1,2,1)}^{\left(3,3,1^{a-4}\right)}+(-1)^{a-4} \chi_{(a-1,2,1)}^{(a+2)}=(-1)^{a-3} \chi_{(2,1)}^{(3)}+(-1)^{a}=0
$$

it follows that $(a, a-1,2,1)$ is a sign partition.

Theorem 4.4. If $a \geqslant 5$ then $(a, a-1,3,1)$ is a sign partition.

Proof. If $a=5$ then $(a, a-1,3,1)=(5,4,3,1)$ and by looking at the character table of $S_{13}$ we can easily check that this is a sign partition. So assume now that $a \geqslant 6$. As $(a-1,3,1)$ is a sign partition for $a \geqslant 6$ from Lemma 1.5 , from Lemma 4.1 we only need to check that $\chi_{(a, a-1,3,1)}^{\beta} \in\{0, \pm 1\}$ for partitions $\beta$ of $2 a+3$ with two $a$-hooks and such that if $\mu$ and $\nu$ are the partitions obtained from $\beta$ by removing an $a$-hook then $\mu$ and $\nu$ have both an $a$-hook and an $(a-1)$-hook.

So let $\beta$ have two $a$-hook. Then $\beta_{(a)}$ is $(3),(2,1)$ or $\left(1^{3}\right)$. Similarly to the previous theorem we will assume that $\beta_{(a)}$ is either $(3)$ or $(2,1)$.

Assume first that $\beta_{(a)}=(3)$. Then, as $\mu$ and $\nu$ can be obtained by adding an $a$-hook to (3) and as there exists exactly $a$ such partitions since $a>3$,

$$
\mu, \nu \in\left\{(a+3),\left(3,3,1^{a-3}\right),\left(3,2,1^{a-2}\right),\left(3,1^{a}\right)\right\} \cup\left\{\left(a-i, 4,1^{i-1}\right): 1 \leqslant i \leqslant a-4\right\} .
$$

As $\mu$ and $\nu$ also have an $(a-1)$-hook it then follows that

$$
\mu, \nu \in\left\{(a+3),\left(3,1^{a}\right),(a-1,4),\left(4,4,1^{a-5}\right)\right\} .
$$

As $a \geqslant 6$

$$
\begin{aligned}
& \chi_{(a-1,3,1)}^{\left(3,1^{a}\right)}=(-1)^{a-2} \chi_{(3,1)}^{(3,1)}=0, \\
& \chi_{(a-1,3,1)}^{(a-1,4)}=-\chi^{(3,1)}=0
\end{aligned}
$$

and so, from Lemma 4.1, we can assume that $\{\gamma, \delta\}=\left\{(a+3),\left(4,4,1^{a-5}\right)\right\}$, that is that $\beta=\left(a+3,5,1^{a-5}\right)$ and then

$$
\chi_{(a, a-1,3,1)}^{\beta}=-\chi_{(a-1,3,1)}^{\left(4,4,1^{a-5}\right)}+(-1)^{a-5} \chi_{(a-1,3,1)}^{(a+3)}=(-1)^{a-4} \chi_{(3,1)}^{(4)}+(-1)^{a-5}=0 .
$$

Assume now that $\beta_{(a)}=(2,1)$. Also in this case, as $a>3$, there exist exactly $a$ partitions which can be obtained by adding an $a$-hook to $(2,1)$ and $\mu$ and $\nu$ are two of them. So

$$
\mu, \nu \in\left\{(a+2,1),(a, 3),\left(2,2,2,1^{a-3}\right),\left(2,1^{a+1}\right)\right\} \cup\left\{\left(a-i, 3,2,1^{i-2}\right): 2 \leqslant i \leqslant a-3\right\} .
$$


As $\mu$ and $\nu$ have an $(a-1)$-hook it follows that

$$
\mu, \nu \in\left\{(a+2,1),(a, 3),\left(2,2,2,1^{a-3}\right),\left(2,1^{a+1}\right),(a-2,3,2),\left(3,3,2,1^{a-5}\right)\right\} .
$$

Since $a \geqslant 6$

$$
\begin{aligned}
\chi_{(a-1,3,1)}^{(a+2,1)} & =\chi_{(3,1)}^{(3,1)}=0, \\
\chi_{(a-1,3,1)}^{\left(2,1^{a+1}\right.} & =(-1)^{a-2} \chi_{(3,1)}^{(2,1,1)}=0 \\
\chi_{(a-1,3,1)}^{(a-2,3)} & =\chi_{(3,1)}^{(2,1,1)}=0, \\
\chi_{(a-1,3,1)}^{\left(3,3,2,1^{a-5}\right)} & =(-1)^{a-4} \chi_{(3,1)}^{(3,1)}=0
\end{aligned}
$$

we again only need to consider one partition $\beta$. In this case $\{\mu, \nu\}=\left\{(a, 3),\left(2,2,2,1^{a-3}\right)\right\}$ and then $\beta=\left(a, 3,3,1^{a-3}\right)$. As

$$
\chi_{(a, a-1,3,1)}^{\left(a, 3,3,1^{a-3}\right)}=\chi_{(a-1,3,1)}^{\left(2,2,2,1^{a-3}\right)}+(-1)^{a-3} \chi_{(a-1,3,1)}^{(a, 3)}=(-1)^{a-3} \chi_{(3,1)}^{(2,2)}+(-1)^{a-2} \chi_{(3,1)}^{(2,2)}=0,
$$

it follows that $(a, a-1,3,1)$ is a sign partition also for $a \geqslant 6$.

\section{Proof of Theorem 1.3}

For $r \leqslant 2$ Theorem 1.3 follows from Lemmas 1.4 and 1.5. So assume now that $r \geqslant 3$.

From Lemma 1.5 and Section 4 it easily follows that if $\gamma \in$ Sign then $\gamma$ is a sign partition.

Assume now that $\gamma=\left(\gamma_{1}, \ldots, \gamma_{r}\right)$ is a sign partition. From Lemma 1.4 it follows that $\left(\gamma_{r-1}, \gamma_{r}\right) \in$ Sign. Also from Lemma 1.5, $\gamma_{i-1}>\gamma_{i}$ for $2 \leqslant i \leqslant r-1$. Fix $2 \leqslant i \leqslant r-1$ and assume that $\left(\gamma_{i}, \ldots, \gamma_{r}\right) \in$ Sign.

Assume that $\left(\gamma_{i-1}, \ldots, \gamma_{r}\right) \neq(5,4,3,2,1)$ and that $\left(\gamma_{i-1}, \ldots, \gamma_{r}\right) \notin$ Sign. From Theorem 1.6 we can find $\beta$ such that $\chi_{\left(\gamma_{i-1}, \ldots, \gamma_{r}\right)}^{\beta} \notin\{0, \pm 1\}$ and $h_{2,1}^{\beta}=\gamma_{i-1}$. Let

$$
\delta:=\left(\beta_{1}+\gamma_{1}+\cdots+\gamma_{i-2}, \beta_{2}, \beta_{3}, \ldots\right) .
$$

Then $\delta$ is a partition of $|\gamma|$. If $i-1=1$ then

$$
\chi_{\gamma}^{\delta}=\chi_{\left(\gamma_{i-1}, \ldots, \gamma_{r}\right)}^{\beta} \notin\{0, \pm 1\},
$$

in contradiction to $\gamma$ being a sign partition. If $i-1 \geqslant 2$ then $\left(1, \beta_{1}+1\right) \in[\delta]$ and

$$
h_{1, \beta_{1}+1}^{\delta}=\gamma_{1}+\cdots+\gamma_{i-2} .
$$

Since $\beta_{2}<\beta_{1}+1$ and $h_{2,1}^{\delta}=h_{2,1}^{\beta}=\gamma_{i-1}<\gamma_{j}$ for $j \leqslant i-2$, we have that also in this case

$$
\chi_{\gamma}^{\delta}=\chi_{\left(\gamma_{i-1}, \ldots, \gamma_{r}\right)}^{\beta} \notin\{0, \pm 1\},
$$

which again gives a contradiction. 
Assume now that $\left(\gamma_{i-1}, \ldots, \gamma_{r}\right)=(5,4,3,2,1)$. If $i-1=1$ or $i-1 \geqslant 2$ and $\gamma_{i-2} \geqslant 7$, then similarly to the previous case

$$
\chi_{\gamma}^{\left(4+\gamma_{1}+\cdots+\gamma_{i-2}, 4,4,3\right)}=\chi_{(5,4,3,2,1)}^{(4,4,4,3)}=-2 .
$$

If $i-1 \geqslant 2$ and $\gamma_{i-1}=6$ we have similarly that

$$
\chi_{\gamma}^{\left(15+\gamma_{1}+\cdots+\gamma_{i-3}, 2,1,1,1,1\right)}=\chi_{(6,5,4,3,2,1)}^{(15,2,1,1,1,1)}=2 .
$$

In either case we have a contradiction with $\gamma$ being a sign partition.

So $\left(\gamma_{i-1}, \ldots, \gamma_{r}\right) \in$ Sign. By induction $\gamma \in$ Sign and so Theorem 1.3 is proved.

\section{Acknowledgements}

Part of the work contained in this paper is contained in the author's master thesis [2], which was written at the University of Copenhagen, under the supervision of Jørn B. Olsson, whom the author thanks for reviewing the paper.

\section{References}

[1] G. James, A. Kerber. The Representation Theory of the Symmetric Group. AddisonWesley Publishing Company, 1981.

[2] L. Morotti. On p-vanishing and sign classes of the symmetric group, Applications of the Murnaghan-Nakayama Formula. Master thesis, Department of Mathematical Sciences, University of Copenhagen (2011).

[3] J. B. Olsson. Combinatorics and Representations of Finite Groups. Vorlesungen aus dem Fachbereich Mathematik der Univerität GH Essen, 1994. Heft 20.

[4] J. B. Olsson. Sign conjugacy classes in symmetric groups. Journal of Algebra 322 (2009):2793-2800. 